\title{
The XMM-Newton wide-field survey in the COSMOS field
}

\section{The point-like X-ray source catalogue ${ }^{\star}, \star \star$}

\author{
N. Cappelluti ${ }^{1,2}$, M. Brusa ${ }^{1,2}$, G. Hasinger ${ }^{1}$, A. Comastri ${ }^{3}$, G. Zamorani ${ }^{3}$, A. Finoguenov ${ }^{1,2}$, R. Gilli $^{3}$, S. Puccetti ${ }^{4}$, \\ T. Miyaji ${ }^{5,16}$, M. Salvato ${ }^{6}$, C. Vignali ${ }^{7}$, T. Aldcroft ${ }^{8}$, H. Böhringer ${ }^{1}$, H. Brunner ${ }^{1}$, F. Civano ${ }^{8}$, M. Elvis ${ }^{8}$, F. Fiore ${ }^{9}$, \\ A. Fruscione ${ }^{8}$, R. E. Griffiths ${ }^{10}$, L. Guzzo ${ }^{11}$, A. Iovino ${ }^{11}$, A. M. Koekemoer ${ }^{12}$, V. Mainieri ${ }^{13}$, N. Z. Scoville ${ }^{6}$, \\ P. Shopbell ${ }^{6}$, J. Silverman ${ }^{14}$, and C. M. Urry ${ }^{15}$
}

(Affiliations can be found after the references)

Received 13 August 2008 / Accepted 14 January 2009

\begin{abstract}
Context. The COSMOS survey is a multiwavelength survey aimed to study the evolution of galaxies, AGN and large scale structures. Within this survey XMM-COSMOS a powerful tool to detect AGN and galaxy clusters. The XMM-COSMOS is a deep X-ray survey over the full 2 deg ${ }^{2}$ of the COSMOS area. It consists of $55 \mathrm{XMM}-$ Newton pointings for a total exposure of $\sim 1.5 \mathrm{Ms}$ with an average vignetting-corrected depth of $40 \mathrm{ks}$ across the field of view and a sky coverage of $2.13 \mathrm{deg}^{2}$.

Aims. We present the catalogue of point-like X-ray sources detected with the EPIC CCD cameras, $\operatorname{the} \log N-\log S$ relations and the X-ray colour-colour diagrams.

Methods. The analysis was performed using the XMM-SAS data analysis package in the $0.5-2 \mathrm{keV}, 2-10 \mathrm{keV}$ and $5-10 \mathrm{keV}$ energy bands. Source detection has been performed using a maximum likelihood technique especially designed for raster scan surveys. The completeness of the catalogue as well as $\log N-\log S$ and source density maps have been calibrated using Monte Carlo simulations.

Results. The catalogs contains a total of 1887 unique sources detected in at least one band with likelihood parameter det_ml $>10$. The survey, which shows unprecedented homogeneity, has a flux limit of $\sim 1.7 \times 10^{-15} \mathrm{erg} \mathrm{cm}^{-2} \mathrm{~s}^{-1}, \sim 9.3 \times 10^{-15} \mathrm{erg} \mathrm{cm}^{-2} \mathrm{~s}^{-1}$ and $\sim 1.3 \times 10^{-14} \mathrm{erg} \mathrm{cm}^{-2} \mathrm{~s}^{-1}$ over $90 \%$ of the area $\left(1.92 \mathrm{deg}^{2}\right)$ in the $0.5-2 \mathrm{keV}, 2-10 \mathrm{keV}$ and $5-10 \mathrm{keV}$ energy band, respectively. Thanks to the rather homogeneous exposure over a large area, the derived $\log N-\log S$ relations are very well determined over the flux range sampled by XMM-COSMOS. These relations have been compared with XRB synthesis models, which reproduce the observations with an agreement of $\sim 10 \%$ in the 5-10 keV and 2-10 keV band, while in the $0.5-2 \mathrm{keV}$ band the agreement is of the order of $\sim 20 \%$. The hard X-ray colors confirmed that the majority of the extragalactic sources in a bright subsample are actually type I or type II AGN. About $20 \%$ of the sources have a X-ray luminosity typical of $\mathrm{AGN}\left(L_{\mathrm{X}}>10^{42} \mathrm{erg} / \mathrm{s}\right)$ although they do not show any clear signature of nuclear activity in the optical spectrum.
\end{abstract}

Key words. galaxies: active - large-scale structure of Universe - X-rays: diffuse background - X-rays: galaxies

\section{Introduction}

The Cosmic evolution survey (COSMOS, Scoville et al. 2007) with its $2 \mathrm{deg}^{2}$ of multiwavelength data is an exceptional laboratory to study active galactic nuclei (AGN), galaxies, large scale structures of the Universe and their co-evolution. The survey uses multi-wavelength imaging and spectroscopy from X-ray to radio wavelengths, including HST, Spitzer and GALEX imaging. The size of the survey has been chosen to sample large-scale structures with linear sizes of $\sim 50 \mathrm{Mpc} \mathrm{h}^{-1}$ at $z=1$ with highly reduced "cosmic" or sample variance.

\footnotetext{
* Based on observations obtained with XMM-Newton, an ESA science mission with instruments and contributions directly funded by ESA Member States and NASA. Based on observations obtained with MegaPrime/MegaCam, a joint project of CFHT and CEA/DAPNIA, at the Canada-France-Hawaii Telescope (CFHT) which is operated by the National Research Council (NRC) of Canada, the Institut National des Sciences de l'Univers of the Centre National de la Recherche Scientifique (CNRS) of France, and the University of Hawaii. This work is based in part on data products produced at TERAPIX.

$\star \star$ Full Table 3 is only available in electronic form at the CDS via anonymous ftp to cdsarc.u-strasbg.fr $(130.79 .128 .5)$ or via http://cdsweb.u-strasbg.fr/cgi-bin/qcat?]/A+A/497/635
}

During the AO3-AO4 and AO6 cycles, XMM-Newton surveyed $2.13 \mathrm{deg}^{2}$ of sky in the COSMOS field in the $0.5-10 \mathrm{keV}$ energy band. The total exposure was $\sim 1.5 \mathrm{Ms}$ split over 55 EPIC pointings. The average resulting exposure over the field of view is $\sim 68 \mathrm{ks}$. The central $0.9 \mathrm{deg}^{2}$ of the COSMOS field also has been observed in X-rays with Chandra for a total of $1.8 \mathrm{Ms}$ by Elvis et al. (2009, hereinafter C-COSMOS).

In this paper we present the X-ray pointlike source catalogue of the 1.5 Ms XMM-COSMOS survey together with the observation diary, data products, $\log N-\log S$ relations and colour-colour plots. A subsample of the first year of XMMCOSMOS data has been presented in Cappelluti et al. (2007a, hereafter Paper II) together with a detailed overview of the data analysis techniques. Here we present data of all the observing cycles, with improved source positioning, higher counting statistics and more precise X-ray photometry.

Optical identifications of XMM-COSMOS sources, performed by taking advantage of the precise source positioning achieved with the complementary Chandra observations, will be presented in another paper (Brusa et al. 2009).

The combination of the moderately deep flux limit and the wide effective area (flux limit of $\sim 1.7 \times 10^{-15} \mathrm{erg} \mathrm{cm}^{-2} \mathrm{~s}^{-1}$ in the 
0.5-2 keV band over $1.92 \mathrm{deg}^{2}$ ) of the XMM-COSMOS made possible the compilation of a sample of sources with low influence of the so called sample or "cosmic" variance. Indeed, in Paper II, assuming these survey parameters, we estimated that in XMM-COSMOS the fluctuations of the source density due to cosmic variance are $<5 \%$. Furthermore, the tiling of the observations was chosen to maximize the uniformity of the sensitivity over a large area of the field.

These particular characteristics, together with the multitude of multiwavelength information available, were designed ad hoc to study the large scale structures traced by X-ray emitting objects like AGN and galaxy clusters and their co-evolution (see e.g. Cappi et al. 2001; Cappelluti et al. 2005, 2007b; Branchesi et al. 2007; Kocevski et al. 2008). In addition these characteristics make the survey sensitive enough to study the evolution of super-massive black holes in the Universe up to high$z$. Considering the high throughput of XMM-Newton at high energies, XMM-COSMOS will provide a valuable sample of absorbed sources to test X-ray background (XRB) synthesis model predictions. Moreover, to understand the nature of the $\mathrm{XRB}$ sources, it is very important to have a detailed, cosmic variance free, measurement of the amplitude of the $\log N-$ $\log S$ relations in several energy bands. It is also worth noting that XMM-COSMOS samples with good accuracy the flux range where most of the XRB flux is produced (i.e. around $\left.\mathrm{S}(2-10 \mathrm{keV}) \sim 10^{-14} \mathrm{erg} \mathrm{cm}^{-2} \mathrm{~s}^{-1}\right)$. Therefore, among the medium-deep X-ray surveys (Brandt \& Hasinger 2005), XMMCOSMOS has the best combination of these characteristics to achieve the goals mentioned above.

The XMM-COSMOS survey, with its large area and counting statistics, provides a large sample of bright sources where the hardness ratio can be measured with good precision. Thanks also to the large amount of spectroscopic data in the field it is possible to compare, in a reliable way, the optical properties with the X-ray properties derived from the hardness ratio analysis for large samples of sources. This is particularly important for AGN classification into absorbed (type II) and unabsorbed (type I). In recent years it was realized (Szokoly et al. 2004) that the classifications based on optical spectroscopy may be affected by strong biases and AGN can be missed or not recognized as such.

The paper is organized as follows: in Sect. 2 we present the observations and we summarize the data reduction techniques; in Sect. 3.1 we report on the source detection; in Sect. 3.2 we present the pointlike source catalog; in Sect. 3.3 we quantify, using Monte Carlo simulations, the completeness of the catalogue; in Sect. 4 we present the $\log N-\log S$ relations; in Sect. 5 we give an overview of the source content of the field using $\mathrm{X}$-ray colour-colour diagrams and the overall results are summarized in Sect. 6. Unless otherwise stated, errors are given at the $1 \sigma$ level and we assume a $\Lambda$ dominated Universe with $H_{0}=70 \mathrm{~km} \mathrm{~s}^{-1} / \mathrm{Mpc}, \Omega_{\mathrm{m}}=0.3$ and $\Omega_{\Lambda}=0.7$.

\section{Observations and data reduction}

The XMM-COSMOS survey covers $2.13 \mathrm{deg}^{2}$ in the equatorial sky in a region bounded by $9^{\mathrm{h}} 57.5^{\mathrm{m}}<\alpha<10^{\mathrm{h}} 03.5^{\mathrm{m}}$ and $1^{\circ} 27.5^{\prime}<\delta<2^{\circ} 57.5^{\prime}$. X-ray observations were performed during XMM-Newton AO3-AO4 from December 2003 to June 2006. The survey consists of a matrix of $5 \times 5$ pointings shifted by $15^{\prime}$ with respect to each other. The matrix of pointings was observed in $\mathrm{AO} 3$ and repeated with a rigid shift of 1' in AO4. The shift was applied to smooth sensitivity drops introduced by the CCD gaps. In Table 1 we present the log of the 55 XMMNewton observations of the COSMOS field.

Because of charged particle flares, two pointings were completely lost, namely $16 \mathrm{~A}$ and $25 \mathrm{~A}$. The lost times were compensated for by tuning the exposures in AO4. Additionally, two pointings (i.e. field 20C and 23C) were re-observed in XMMNewton AO6 (May 2007) for $32 \mathrm{ks}$ each to compensate for time losses. At the time of writing no further observing campaigns of the COSMOS field are planned with XMM-Newton.

In Paper II we analyzed a first sample of 23 fields observed with XMM-Newton during AO3 labeled in Table 1. The total exposure was $\sim 504 \mathrm{ks}$ after the cleaning of the particle background flares. The faintest sources in the field have a flux of $7 \times 10^{-16} \mathrm{erg} \mathrm{cm}^{-2} \mathrm{~s}^{-1}, 4 \times 10^{-15} \mathrm{erg} \mathrm{cm}^{-2} \mathrm{~s}^{-1}$ and $9 \times 10^{-15}$ erg $\mathrm{cm}^{-2} \mathrm{~s}^{-1}$ in the $0.5-2 \mathrm{keV}, 2-10 \mathrm{keV}$ and $5-10 \mathrm{keV}$ energy bands, respectively, while a flux limit of $\sim 1.7 \times 10^{-15}$ erg $\mathrm{cm}^{-2} \mathrm{~s}^{-1}, \sim 9.3 \times 10^{-15} \mathrm{erg} \mathrm{cm}^{-2} \mathrm{~s}^{-1}$ and $\sim 1.3 \times 10^{-14}$ $\operatorname{erg~cm} \mathrm{cm}^{-2} \mathrm{~s}^{-1}$ was achieved over $90 \%$ of the area $\left(1.92 \mathrm{deg}^{2}\right)$ in the $0.5-2 \mathrm{keV}, 2-10 \mathrm{keV}$ and 5-10 keV energy band, respectively. The preliminary catalogue based on those data consisted of 1390 independent sources and 1281, 784 and 186 source in the three bands, respectively. We used that catalog to produce the first XMM-COSMOS $\log N-\log S$ relations as well as the first study of the cosmic or sample variance in X-ray surveys. Paper II also contains a detailed section on data analysis techniques, including event cleaning, image processing, astrometry, source detection and Monte Carlo simulations. In this section we briefly summarize the analysis method; we refer the reader to Paper II for a detailed description.

XMM-Newton was operated in imaging mode using the EPIC CCD cameras in full frame mode. X-ray event files were searched for particle background flares and screened with the technique described in Paper II. In order to reduce the instrumental background, the energy channels between $1.45 \mathrm{keV}$ and $1.54 \mathrm{keV}$ were discarded in both the MOS and PN data. To remove the strong $\mathrm{Cu}$ fluorescence features in the PN background we also discarded the energy bands $7.2 \mathrm{keV}-7.6 \mathrm{keV}$ and $7.8 \mathrm{keV}-8.2 \mathrm{keV}$. The total scheduled EPIC exposure time was $1464 \mathrm{ks}$ while, after the background cleaning the sum of the PN good time intervals (GTI) was $\sim 988 \mathrm{ks}$ and $1207 \mathrm{ks}$ for both MOS1 and MOS2.

Due to the slow decrease of the solar activity from its maximum in 2000 to its minimum in 2007 (Hathaway et al. 1999), observations performed in $\mathrm{AO} 3$ and in the first part of A04 have a significantly higher background level than in the second part of $\mathrm{AO} 4$ and the two observations in AO6. Event files were processed using the XMM-Newton standard analysis software (SAS) version 6.7.0. After the removal of high background intervals we searched for and removed hot/dead columns and pixels. Images were created in the $0.5-2 \mathrm{keV}, 2-8 \mathrm{keV}$ and $4.5-10 \mathrm{keV}$ energy bands. In the same bands we created spectral weighted exposure maps assuming a power-law model with photon spectral index $\Gamma=2$ in the $0.5-2 \mathrm{keV}$ band and $\Gamma=1.7$ in the $2-8 \mathrm{keV}$ and $4.5-10 \mathrm{keV}$ bands.

The $0.5-2 \mathrm{keV}$ exposure map of the XMM-COSMOS survey is shown in Fig. 1, while in Fig. 2 we show a false colour X-ray image of the entire field.

In order to compute background maps, we performed a preliminary source detection using a sliding cell technique. Using a threshold of $2.5 \sigma$ with the XMMSAS software "eboxdetect", we excised all the detected sources from all the images. The resulting images were fitted with a double component model (a flat and a vignetted component) to mimic the particle and the X-ray sky background. 
Table 1. The XMM-Newton observation log of the XMM-COSMOS survey.

\begin{tabular}{|c|c|c|c|c|c|c|c|c|}
\hline ID & Revolution & OBS_ID & $\begin{array}{c}\text { Date } \\
\text { YYYY-MM-DDTHH:MM:SS }\end{array}$ & $\begin{array}{c}\text { RA } \\
\mathrm{hm} \mathrm{s}\end{array}$ & $\begin{array}{l}\text { Dec } \\
0, \prime \prime \prime\end{array}$ & $\begin{array}{l}\text { Exposure } \\
\text { ks }\end{array}$ & $\begin{array}{l}\text { GTI PN } \\
\text { ks }\end{array}$ & $\begin{array}{c}\text { GTI MOS } \\
\text { ks }\end{array}$ \\
\hline $1 \mathrm{~A}^{a}$ & 917 & 0203360101 & 2004-12-11T13:23:10 & 100226.4 & 24236.0 & 30.8 & 28.8 & 30.5 \\
\hline 1B & 1090 & 0302350101 & 2005-11-21T20:57:04 & 100226.4 & 24336.0 & 19.9 & 14.2 & 17.8 \\
\hline $2 \mathrm{~A}^{a}$ & 917 & 0203360201 & 2004-12-11T22:36:27 & 100226.4 & 22736.0 & 44.1 & 13.7 & 15.9 \\
\hline 2B & 1190 & 0302350201 & 2006-06-08Т15:28:17 & 100230.4 & 22736.0 & 19.9 & 12.5 & 16.1 \\
\hline $3 \mathrm{~A}^{a}$ & 994 & 0203360301 & $2005-05-14 \mathrm{~T} 03: 18: 03$ & 100226.4 & 21236.0 & 32.2 & 30.1 & 31.9 \\
\hline 3B & 1083 & 0302350301 & 2005-11-07T08:41:36 & 100226.4 & 21336.0 & 5.3 & 1.6 & 4.3 \\
\hline $3 \mathrm{C}$ & 1186 & 0302353101 & 2006-06-01T09:43:42 & 100226.4 & 21336.0 & 20.8 & 16.5 & 19.0 \\
\hline $4 \mathrm{~A}^{a}$ & 907 & 0203360401 & 2004-11-21T05:12:10 & 100226.4 & 15736.0 & 30.8 & 25.6 & 29.1 \\
\hline 4B & 1090 & 0302350401 & 2005-11-22Т03:07:03 & 100230.4 & 15736.0 & 25.1 & 7.3 & 11.6 \\
\hline $5 \mathrm{~A}^{a}$ & 907 & 0203360501 & 2004-11-21T14:25:29 & 100226.4 & 14236.0 & 30.8 & 26.1 & 29.0 \\
\hline $5 \mathrm{~B}$ & 1089 & 0302350501 & 2005-11-19T16:32:05 & 100226.4 & 14336.0 & 19.9 & 17.9 & 19.7 \\
\hline $6 \mathrm{~A}^{a}$ & 819 & 0203360601 & 2004-05-30T00:49:22 & 100126.4 & 24236.0 & 30.8 & 22.1 & 24.8 \\
\hline $6 \mathrm{~B}$ & 1186 & 0302350601 & 2006-06-01T03:33:43 & 100122.4 & 24236.0 & 19.9 & 15.4 & 18.8 \\
\hline $7 \mathrm{~A}^{a}$ & 731 & 0203360701 & 2003-12-06T01:35:44 & 100126.4 & 22736.0 & 34.4 & 31.9 & 34.2 \\
\hline $7 \mathrm{~B}$ & 1091 & 0302350701 & 2005-11-23T05:06:10 & 100126.4 & 22836.0 & 19.9 & 17.8 & 19.1 \\
\hline $8 \mathrm{~A}^{a}$ & 905 & 0203360801 & $2004-11-17 \mathrm{~T} 21: 49: 38$ & 100126.4 & 21236.0 & 53.0 & 26.8 & 36.5 \\
\hline $8 \mathrm{~B}$ & 1092 & 0302350801 & 2005-11-25T19:44:36 & 100122.4 & 21236.0 & 19.9 & 17.6 & 19.4 \\
\hline $9 \mathrm{~A}^{a}$ & 906 & 0203360901 & 2004-11-20T00:46:35 & 100126.4 & 15736.0 & 36.2 & 20.8 & 23.9 \\
\hline $9 \mathrm{~B}$ & 1095 & 0302350901 & $2005-12-02 \mathrm{~T} 02: 52: 30$ & 100126.4 & 15836.0 & 24.4 & 11.0 & 11.8 \\
\hline $9 \mathrm{C}$ & 1179 & 0302353001 & 2006-05-18T12:17:32 & 100126.4 & 15836.0 & 9.9 & 2.4 & 4.5 \\
\hline $10 \mathrm{~A}^{a}$ & 907 & 0203361001 & $2004-11-21 \mathrm{~T} 23: 38: 52$ & 100126.4 & 14236.0 & 45.5 & 12.9 & 17.2 \\
\hline $10 \mathrm{~B}$ & 1088 & 0302351001 & 2005-11-17T04:04:51 & 100122.4 & 14236.0 & 43.5 & 37.2 & 42.8 \\
\hline $11 \mathrm{~A}^{a}$ & 912 & 0203361101 & $2004-12-01 \mathrm{~T} 23: 23: 41$ & 100026.4 & 24236.0 & 44.2 & 19.5 & 22.8 \\
\hline $11 \mathrm{~B}$ & 1176 & 0302351101 & 2006-05-12Т09:13:47 & 100026.4 & 24336.0 & 45.6 & 16.5 & 18.8 \\
\hline $12 \mathrm{~A}^{a}$ & 732 & 0203361201 & $2003-12-08 \mathrm{~T} 18: 19: 32$ & 100026.4 & 22736.0 & 34.9 & 25.1 & 26.6 \\
\hline $12 \mathrm{~B}$ & 1091 & 0302351201 & 2005-11-23T11:16:10 & 100030.4 & 22736.0 & 19.9 & 13.9 & 15.8 \\
\hline $13 \mathrm{~A}^{a}$ & 733 & 0203361301 & 2003-12-10T11:23:58 & 100026.4 & 21236.0 & 31.8 & 25.3 & 26.5 \\
\hline 13B & 1091 & 0302351301 & 2005-11-23Т17:26:09 & 100026.4 & 21336.0 & 19.9 & 18.0 & 19.2 \\
\hline $14 \mathrm{~A}^{a}$ & 733 & 0203361401 & 2003-12-10T01:52:22 & 100026.4 & 15736.0 & 32.0 & 30.1 & 31.1 \\
\hline 14B & 1182 & 0302351401 & 2006-05-24T03:48:33 & 100030.4 & 15736.0 & 23.0 & 10.4 & 19.4 \\
\hline $15 \mathrm{~A}^{a}$ & 906 & 0203361501 & 2004-11-19T15:33:15 & 100026.4 & 14236.0 & 30.9 & 20.2 & 26.9 \\
\hline 15B & 1179 & 0302351501 & 2006-05-18Т06:07:33 & 100026.4 & 14336.0 & 19.9 & 12.6 & 16.0 \\
\hline $16 \mathrm{~A}^{a}$ & 914 & 0203361601 & 2004-12-05T23:28:32 & 095926.4 & 24236.0 & 41.1 & 0.0 & 0.0 \\
\hline $16 \mathrm{~B}$ & 1093 & 0302351601 & 2005-11-27T17:59:55 & 095922.4 & 24236.0 & 57.3 & 28.2 & 36.6 \\
\hline $17 \mathrm{~A}^{a}$ & 917 & 0203361701 & 2004-12-11Т03:53:07 & 095926.4 & 22736.0 & 31.9 & 29.9 & 31.4 \\
\hline 17B & 1179 & 0302351701 & $2006-05-17 \mathrm{~T} 23: 57: 32$ & 095926.4 & 22836.0 & 19.9 & 17.7 & 19.6 \\
\hline $18 \mathrm{~A}^{a}$ & 734 & 0203361801 & 2003-12-11T22:33:13 & 095926.4 & 21236.0 & 28.9 & 26.2 & 27.7 \\
\hline $18 \mathrm{~B}$ & 1179 & 0302351801 & $2006-05-17 \mathrm{~T} 17: 47: 33$ & 095922.4 & 21236.0 & 19.9 & 16.8 & 18.6 \\
\hline $19 \mathrm{~A}^{a}$ & 918 & 0203361901 & $2004-12-12 \mathrm{~T} 21: 37: 00$ & 095926.4 & 15736.0 & 30.9 & 23.3 & 25.3 \\
\hline 19B & 1178 & 0302351901 & 2006-05-15T23:34:59 & 095926.4 & 15836.0 & 19.9 & 9.9 & 17.9 \\
\hline $20 \mathrm{~A}^{a}$ & 994 & 0203362001 & 2005-05-14T12:52:14 & 095926.4 & 14236.0 & 31.9 & 7.0 & 9.2 \\
\hline $20 \mathrm{~B}$ & 1178 & 0302352001 & 2006-05-15T17:24:58 & 095922.4 & 14236.0 & 19.9 & 4.9 & 16.6 \\
\hline $20 \mathrm{C}^{b}$ & 1356 & 0501170101 & 2007-05-06T00:23:52 & 095922.4 & 14236.0 & 33.9 & 32.0 & 33.3 \\
\hline $21 \mathrm{~A}^{a}$ & 916 & 0203362101 & 2004-12-09T07:16:01 & 095826.4 & 24236.0 & 62.6 & 60.3 & 61.7 \\
\hline $22 \mathrm{~A}^{a}$ & 898 & 0203362201 & 2004-11-03Т06:02:44 & 095826.4 & 22736.0 & 30.9 & 28.0 & 30.5 \\
\hline $22 \mathrm{~B}$ & 1176 & 0302352201 & 2006-05-11T19:47:08 & 095830.4 & 22736.0 & 21.9 & 7.0 & 10.8 \\
\hline $23 \mathrm{~A}^{a}$ & 992 & 0203362301 & 2005-05-09T19:01:30 & 095826.4 & 21236.0 & 30.9 & 1.3 & 28.1 \\
\hline $23 \mathrm{~B}$ & 1176 & 0302352301 & $2006-05-12 \mathrm{~T} 02: 30: 29$ & 095826.4 & 21336.0 & 21.9 & 4.3 & 7.4 \\
\hline $23 C^{b}$ & 1362 & 0501170201 & 2007-05-18Т03:17:39 & 095826.4 & 21336.0 & 36.0 & 28.1 & 33.9 \\
\hline $24 \mathrm{~A}^{a}$ & 992 & 0203362401 & 2005-05-10T04:14:50 & 095826.4 & 15736.0 & 30.9 & 17.4 & 23.0 \\
\hline $24 \mathrm{~B}$ & 1175 & 0302352401 & 2006-05-09T19:36:30 & 095830.4 & 15736.0 & 24.9 & 0.2 & 21.9 \\
\hline $24 \mathrm{C}$ & 1190 & 0302353201 & 2006-06-09T01:36:12 & 095830.4 & 15736.0 & 19.3 & 9.7 & 14.6 \\
\hline $25 \mathrm{~A}^{a}$ & 992 & 0203362501 & 2005-05-10T13:28:11 & 095826.4 & 24236.0 & 31.9 & 0.0 & 0.0 \\
\hline $25 \mathrm{~B}$ & 1175 & 0302352501 & 2006-05-10Т03:09:54 & 095826.4 & 14336.0 & 24.5 & 22.6 & 23.9 \\
\hline $25 \mathrm{C}$ & 1190 & 0302353301 & 2006-06-09Т07:36:11 & 095826.4 & 14336.0 & 18.9 & 11.6 & 14.3 \\
\hline
\end{tabular}

From left to right: Field ID, revolution, OBS_ID, observation start, right ascension, declination, duration of the exposure, good time interval (GTI) for the PN and MOS camera, respectively; ${ }^{a}$ fields observed in XMM-Newton AO3 presented by Hasinger et al. (2007) and used for Paper II; ${ }^{b}$ fields observed in XMM-Newton AO6.

Astrometry corrections were estimated as in Paper II by cross-correlating highly significant (i.e. det_ml $>15$, see Sect. 3.1) X-ray sources detected in each pointing, with the catalog of galaxies detected in the I-band by CFHT-MEGACAM (McCracken et al. 2007) and computing the most likely shift using the XMM-SAS software "eposcorr". The mean astrometric shift is similar to that reported in Paper II, being $\Delta(\alpha) \sim 1.4^{\prime \prime}$ and $\Delta(\delta) \sim 0.2^{\prime \prime}$.

\section{Source detection and source catalogue}

\subsection{Source detection}

We ran a two steps source detection in three energy bands, i.e. the $0.5-2 \mathrm{keV}, 2-8 \mathrm{keV}$ and $4.5-10 \mathrm{keV}$.

By using the XMM-SAS tool "eboxdetect" we first ran a sliding cell detection to select source candidates in the field. Differently from Paper II, we used the $2-8 \mathrm{keV}$ band in place of 


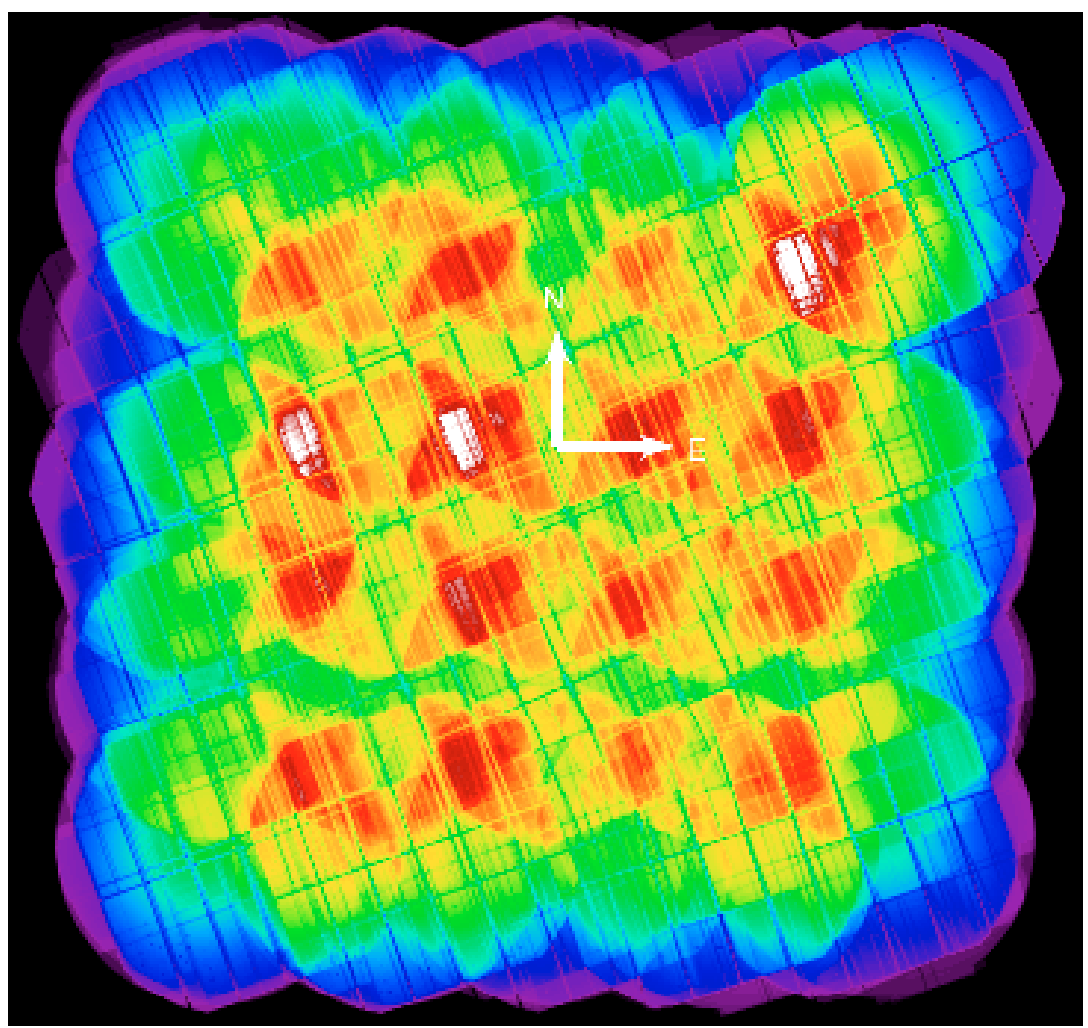

Fig. 1. Colour coded vignetting corrected $0.5-2 \mathrm{keV}$ exposure map of the XMM-COSMOS survey. The maximum effective depth achieved on the field is $\sim 84 \mathrm{ks}$ (white) and the mean exposure is $\sim 68 \mathrm{ks}$ (green).

the $2-4.5 \mathrm{keV}$ band. This because a reanalysis of the AO3 data showed that the $2-8 \mathrm{keV}$ band yields a better estimate of the $2-10 \mathrm{keV}$ source counts (i.e. in the $2-4.5 \mathrm{keV}$ band we detected $10 \%$ fewer sources than in the $2-8 \mathrm{keV}$ band). Moreover we determined that excluding the $8-10 \mathrm{keV}$ events from the analysis slightly enhanced the signal-to-noise ratio of most the $2-10 \mathrm{keV}$ sources. The $4.5-10 \mathrm{keV}$ band has been fully exploited in order to find the most absorbed sources.

If $P$ is the probability that a Poissonian fluctuation of the background is detected as a spurious source, the likelihood of the detection is then defined as det_ml $=-\ln (P)$. All the source candidates with det_ml $<4$ were discarded. Making use of the XMM-SAS tool "emldetect" we then performed a maximum likelihood fit of each source candidate to a PSF model available in the XMM-Newton libraries. All the sources were also fitted with a convolution of a $\beta$-model cluster brightness profile (Cavaliere \& Fusco-Femiano 1976) with the XMM-Newton PSF, to determine a possible extension in the detected signal. A source is classified as extended if the likelihood for the $\beta$-model fit exceeds that of the pointlike case of 10 in det_ml. The sources are fitted simultaneously in the $0.5-2 \mathrm{keV}, 2-8 \mathrm{keV}$ and $4.5-10 \mathrm{keV}$ energy bands and the free parameters of the fit are position, flux and extension.

Moreover, the calculation of the positional uncertainties in each band also makes use of the information in other bands and thus source positioning is extremely accurate in all the energy bands (see also discussion in Paper II). In Fig. 3 we show the distribution of the statistical uncertainties on the source positions in arcsec as output by the emldetect task. The median statistical astrometric uncertainty, including also a systematic error of $0.75^{\prime \prime}$ (see Brusa et al. 2009, for a detailed discussion), is $1.77^{\prime \prime}$. The reliability of the estimated source positions is

\footnotetext{
${ }^{1}$ Similar results also have been obtainedvia Monte Carlo simulations.
}

confirmed by the distribution of the offset between X-ray sources and optical counterparts. Count rates estimated in the $2-8 \mathrm{keV}$ and $4.5-10 \mathrm{keV}$ energy bands were extrapolated into $2-10 \mathrm{keV}$ and $5-10 \mathrm{keV}$ fluxes, respectively. In these bands we computed energy conversion factors (ECF) by assuming a power-law spectrum with spectral index $\Gamma=1.7$ and Galactic column density $N_{\mathrm{H}}=2.5 \times 10^{20} \mathrm{~cm}^{-2}$. In the $0.5-2 \mathrm{keV}$ band, we directly converted the count-rate into fluxes assuming a spectral index $\Gamma=2.0$ and Galactic column density $N_{\mathrm{H}}=2.5 \times 10^{20} \mathrm{~cm}^{-2}$. The choice of these spectral indices is driven by the findings of Mainieri et al. (2007, 2008). They measured an average spectral index $\langle\Gamma\rangle \sim 1.7$ for the XMM-COSMOS sources. In the soft band we have chosen a steeper index to take into account the contribution of the soft excess. Moreover these values of $\Gamma$ are widely used in the literature (see e.g. Hasinger et al. 1993; Baldi et al. 2002) and therefore this choice has also the scope of a better comparison with previous works, especially when comparing the $\log N-\log S$ relations.

The adopted $\mathrm{ECFs}^{2}$ are $10.45 \mathrm{cts} \mathrm{s}^{-1} / 10^{-11} \mathrm{erg} \mathrm{cm}^{-2} \mathrm{~s}^{-1}, 2.06$ cts s$^{-1} / 10^{-11} \mathrm{erg} \mathrm{cm}^{-2} \mathrm{~s}^{-1}$ and $1.21 \mathrm{cts} \mathrm{s}^{-1} / 10^{-11} \mathrm{erg} \mathrm{cm}^{-2} \mathrm{~s}^{-1}$ in the $0.5-2 \mathrm{keV}, 2-8 \mathrm{keV}$ and $4.5-10 \mathrm{keV}$ energy bands, respectively. All the sources with a maximum likelihood parameter det_ml $>10$ in at least one band have been included in the present catalog. This threshold corresponds to a fraction of expected spurious sources of the order of $1.5 \%$ in the $0.5-2 \mathrm{keV}$ band and $\sim 0.5 \%$ in the other energy bands. Since in this work we used a more conservative detection threshold than in Paper II (det_ml $>6$ ), the fraction of spurious sources has been significantly reduced.

Significant detections have been achieved only in a subset of the energy bands. In the bands where the detection is not

\footnotetext{
2 The ECF values also take into account the energy channels discarded to decrease the background.
} 


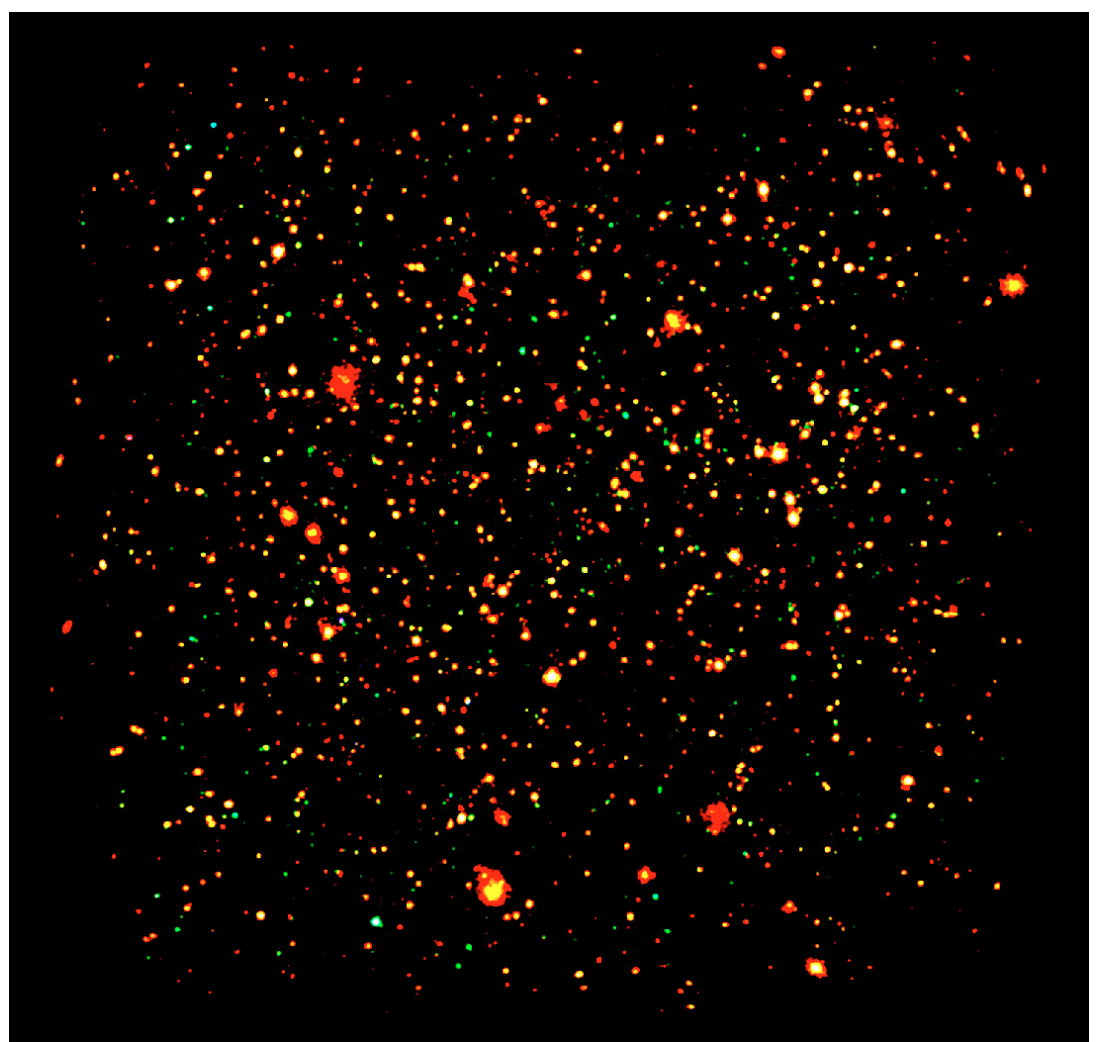

Fig. 2. False colour X-ray image of the COSMOS field: red, green and blue colours represent the $0.5-2 \mathrm{keV}, 2-4.5 \mathrm{keV}$ and $4.5-10 \mathrm{keV}$ energy bands, respectively.

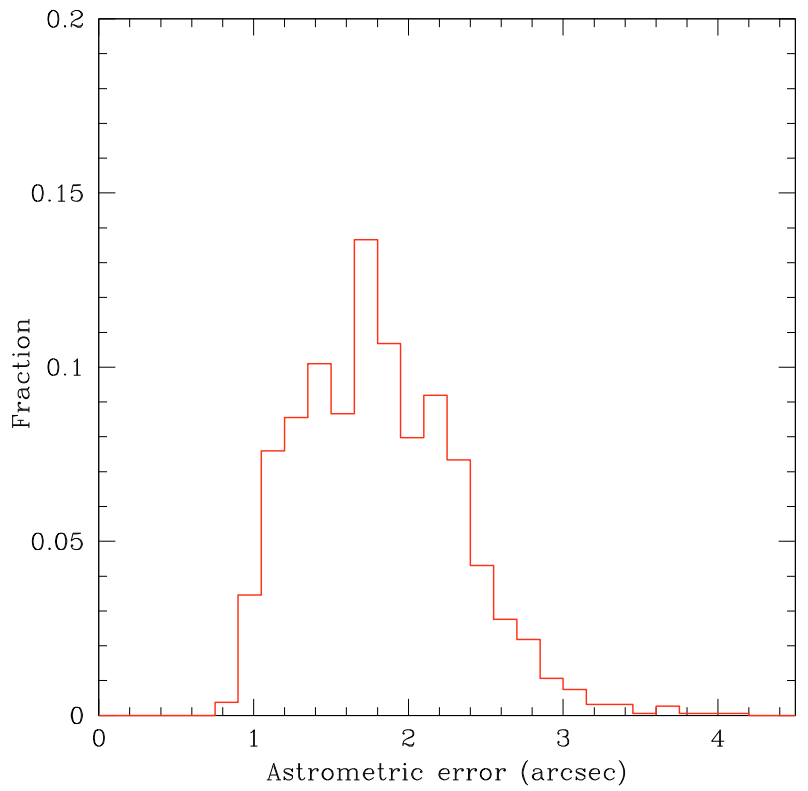

Fig. 3. The distribution of the positional uncertainties of the XMMCOSMOS detections.

significant, we computed $1 \sigma$ upper limits of the counts using the prescriptions of Narsky (2000). Given M counts actually measured in a region of $30^{\prime \prime 3}$ at the position of the source and B background counts (estimated from our background maps), the $1 \sigma$ upper limit is defined as the number of counts $X$ that

\footnotetext{
3 We checked that a $30^{\prime \prime}$ aperture gives the best agreement between aperture photometry and the maximum likelihood PSF fitting technique.
}

gives the probability of observing $\mathrm{M}$ (or fewer) counts equal to the formal $68.3 \%$ Gaussian probability:

$P(\leq M, X+B)=P_{\text {Gauss }}(68.3 \%)$.

Assuming Poissonian statistics, this equation becomes:

$P_{\text {Gauss }}=\mathrm{e}^{-(X+B)} \sum_{i=0}^{M} \frac{(X+B)^{i}}{i !}$.

By solving Eq. (2) iteratively in the case of $P_{\text {Gauss }}=0.683$, we obtained the $1 \sigma$ upper limit X. The upper limits were then converted into count-rates and fluxes by diving by the exposure map and then applying the ECFs. We removed from the catalogue about 20 sources lying close to clear artifacts of the image (i.e. field and pointing boundaries or unremoved hot pixels). With the method described above we selected a total of 1887, independent sources. Each source has been named with a unique ID number. 1621 sources have been detected in the $0.5-2 \mathrm{keV}$ energy band, while 1111 and 251 sources are detected in the $2-10 \mathrm{keV}$ and 5-10 keV band, respectively. The number of sources with a significant detection in only one band is 771 for $0.5-2 \mathrm{keV}$ band, 237 and 5 for the 2-10 keV and 5-10 keV bands, respectively. The faintest sources in the field have fluxes of $5.0 \times 10^{-16}$ erg cm $\mathrm{cs}^{-1}, 2.5 \times 10^{-15} \mathrm{erg} \mathrm{cm}^{-2} \mathrm{~s}^{-1}$ and $5.1 \times 10^{-15} \mathrm{erg} \mathrm{cm}^{-2} \mathrm{~s}^{-1}$ in the three energy bands.

A summary of the source detection results is shown in Table 2.

Thanks to our PSF fitting technique we were able to detect 109 additional extended sources. The catalog of the extended sources, together with a detailed and more extensive analysis of their properties will be presented in a forthcoming paper by Finoguenov et al. (2008). 
Table 2. Summary of the total detections, single band detections, faintest flux limits, flux limits at 50\%, $90 \%$ of the total area and flux limits observable on the full area in the $0.5-2 \mathrm{keV}, 2-10 \mathrm{keV}$ and $5-10 \mathrm{keV}$ energy bands, respectively.

\begin{tabular}{|c|c|c|c|c|c|c|}
\hline Band & Total detections & Single-band detections & $\begin{array}{c}S_{\lim } \\
\text { erg cm}{ }^{-2} \mathrm{~s}^{-1} / 10^{-15}\end{array}$ & $\begin{array}{c}S_{50 \%} \\
\text { erg cm}{ }^{-2} \mathrm{~s}^{-1} / 10^{-15}\end{array}$ & $\begin{array}{c}S_{90 \%} \\
\text { erg cm } \mathrm{cm}^{-2} \mathrm{~s}^{-1} / 10^{-15}\end{array}$ & 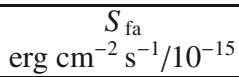 \\
\hline $0.5-2 \mathrm{keV}$ & 1621 & 771 & 0.50 & 1.00 & 1.70 & 3.00 \\
\hline $2-10 \mathrm{keV}$ & 1111 & 237 & 2.50 & 5.60 & 9.30 & 15.00 \\
\hline $5-10 \mathrm{keV}$ & 251 & 5 & 5.10 & 11.00 & 13.0 & 20.00 \\
\hline
\end{tabular}

\subsection{Source catalogue}

In Table 3 we show, as an example, the first 50 entries of the catalogue as they appear on-line. The table is structured as follows: Column 1: IAU Name, Column 2: XID, Column 3: $\alpha$ (deg), Column 4: $\delta$ (deg), Column 5: Positional error (arcsec), Column 6: $0.5-2 \mathrm{keV}$ flux (erg $\mathrm{cm}^{-2} \mathrm{~s}^{-1} / 10^{-14}$ ), Column 7: 0.5-2 keV net counts, Column 8: 0.5-2 keV likelihood parameter det_ml, Column 9: $0.5-2 \mathrm{keV}$ background counts (cts/pix $)^{4}$, Column 10: 0.5-2 keV vignetting corrected exposure (ks), Column 11: $2-10 \mathrm{keV}$ flux (erg $\mathrm{cm}^{-2} \mathrm{~s}^{-1} / 10^{-14}$ ), Column 12: 2-8 keV net counts , Column 13: 2-8 keV likelihood parameter det_ml, Column 14: 2-8 keV background counts (cts/pix), Column 15: 2-8 keV vignetting corrected exposure (ks), Column 16: 5-10 keV flux, (erg $\mathrm{cm}^{-2} \mathrm{~s}^{-1} / 10^{-14}$ ), Column 17: 4.5-10 keV net counts, Column 18: 4.5-10 keV likelihood parameter det_ml, Column 19: 4.5-10 keV background counts, Column 20: 5-10 keV vignetting corrected exposure (ks).

The interactive and machine readable full version of the cata$\log$ can be downloaded at http: //irsa. ipac. caltech. edu/ data/COSMOS/+. For sources with no significant detection in a band, we list upper limits with negative values of flux. In this case, we also quote a value of cts $=0$, det_ml $=-1$ and $\mathrm{Bkg}=-1$ (background) in the band where the detection is not significant.

The flux errors are the statistical uncertainties estimated from the maximum likelihood and do not include uncertainties introduced by the choice of the spectral model to estimate the flux. We determined that by varying by $\Delta \Gamma=0.3$ the spectral index assumed in computing the fluxes, the resulting variation of the flux estimate is of the order of $2 \%, 9 \%$, and $4 \%$ in the three bands.

The Chandra coverage of the inner area of the XMMCOSMOS field (Elvis et al. 2009) offers a unique possibility to investigate the effect of source confusion in our catalog.

The Chandra field covers about half of the XMM-COSMOS field. Of the 1887 XMM-sources with det_ml>10, $946(50.1 \%)$ have been observed by Chandra with an exposure longer than $30 \mathrm{ks}$, and 876 of them are present in the C-COSMOS point-like source catalog (Elvis et al. 2009). Twenty-four of the 876 XMM pointlike sources with Chandra coverage $(2.7 \%)$ are actually resolved into two different Chandra sources, which lie between 2 and 10 arcsec from each other and have been blurred by the XMM large PSF. We then used the Chandra source counterpart positions of these 24 "blended sources" (48 different positions) as the input catalog for emldetect and we fitted these sources keeping the position parameter fixed at the Chandra value. As a result only 2/24 XMM-COSMOS sources have been deblended into 4 XMM-Newton sources, namely XID \#67 and XID \#82, while the remaining 22 sources have been detected again as a single XMM-COSMOS source with properties consistent with

${ }^{4}$ The pixel scale is $4^{\prime \prime} /$ pix.

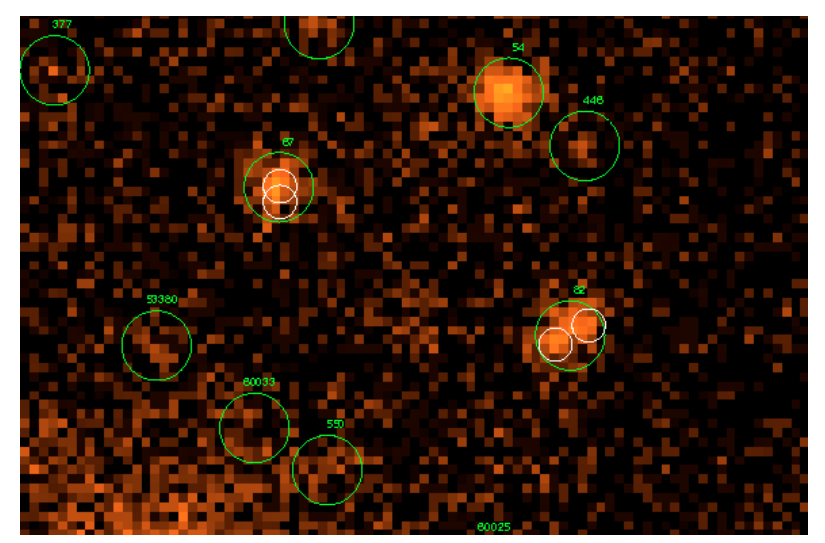

Fig. 4. The region $\left(6^{\prime} \times 3^{\prime}\right)$ containing the sources XID \#67 and \#82. Green circles correspond to the XMM-Newton detections, while white circles correspond to the Chandra detections.

those presented in the catalogue ${ }^{5}$. Therefore we can conclude that our sample contains $<2.7 \%$ of the sources which could be resolved into two sources at the Chandra-COSMOS flux limit. In Fig. 4 we show the XMM-Newton image of a region containing the two deblended sources, which by chance are close to each other.

\subsection{Monte Carlo simulations, sky coverage and sensitivity maps}

In order to estimate the sky coverage of our survey, we performed Monte Carlo simulations as described in Paper II. The precision of the photometry as well as positional accuracy were also discussed in Paper II. Here we give an overview of the procedure adopted for the production of random X-ray sky images and their analysis.

Twenty series of 55 XMM-Newton images were created with the same pattern, exposure maps and background levels as the real data. We produced 20 random input source catalogs with sources randomly placed in the field of view and fluxes distributed according to the AGN $\log N-\log S$ distributions predicted by the Gilli et al. (2007) XRB synthesis model. The input fluxes were converted into count-rates by folding through the response matrices the same spectral model assumed to compute fluxes and to weight exposure maps. The counts of the sources were then convolved with XMM-Newton PSF templates available in the XMM-Newton calibration database and reproduced on the detector. We then applied to the simulated fields the same source detection procedure used in the real data producing 20 independent output catalogs.

The sky coverage is then obtained by dividing the number of detected sources at each flux by the number of input sources and

\footnotetext{
5 We kept these sources as single entries in the catalog for self consistency with our statistical analysis.
} 

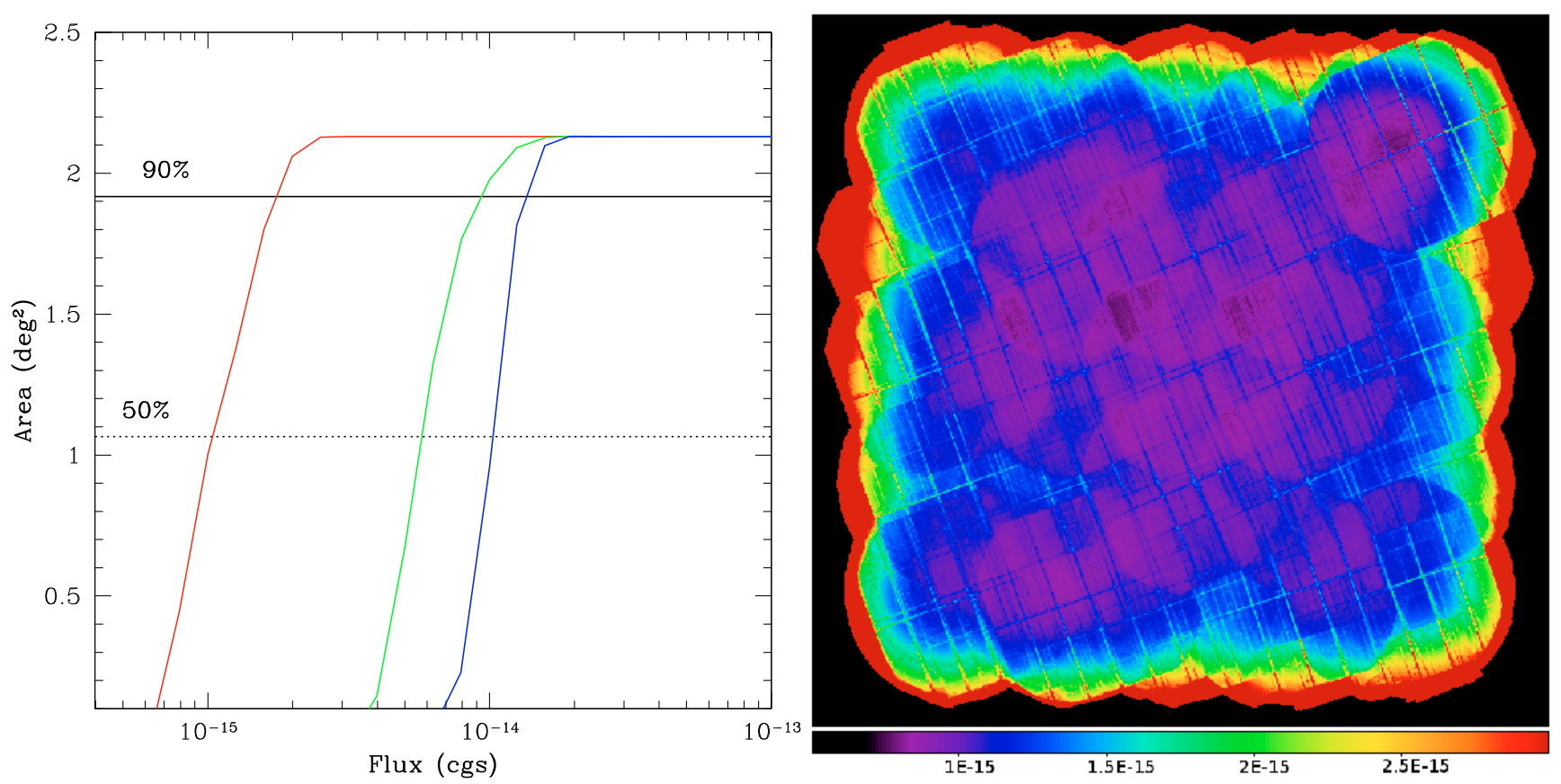

Fig. 5. Left panel: the sky coverage versus flux for the $0.5-2 \mathrm{keV}, 2-10 \mathrm{keV}$ and $5-10 \mathrm{keV}$ bands, represented by red, green and blue solid lines. The horizontal solid and dashed lines show the $90 \%$ and 50\% completeness levels. Right panel: the $0.5-2 \mathrm{keV}$ sensitivity map of XMM-COSMOS in erg cm$~^{-2} \mathrm{~s}^{-1}$. The map is plotted in colour coded scale from $5 \times 10^{-16} \mathrm{erg} \mathrm{cm}^{-2} \mathrm{~s}^{-1}$ (magenta) to $3 \times 10^{-15} \mathrm{erg} \mathrm{cm}^{-2} \mathrm{~s}^{-1}$ (red).

rescaling for a total area of $2.13 \mathrm{deg}^{2}$. By using as a model the Gilli et al. (2007) $\log N-\log S$, it is possible that the simulated $\log N-\log S$ could be slightly different from the real $\log N-$ $\log S$. This could introduce some biases in the estimation of the effective area. However, Schmitt \& Maccacaro (1986) showed that the effect of a different slope of the $\log N-\log S$ is negligible when the threshold of the source detection is higher than 3-4 $\sigma$.

The sky coverage in the three energy bands under investigation is plotted in the left panel of Fig. 5 As a result of the simulations, we obtained that $90 \%$ of the survey area is sensitive to flux limits of $\sim 1.7 \times 10^{-15} \mathrm{erg} \mathrm{cm}^{-2} \mathrm{~s}^{-1}, \sim 9.3 \times 10^{-15}$ erg cm$~^{-2} \mathrm{~s}^{-1}$ and $\sim 1.3 \times 10^{-14} \mathrm{erg} \mathrm{cm}^{-2} \mathrm{~s}^{-1}$ in the three energy bands, respectively. Additionally, we determined that the survey is sensitive, over the full field of view (i.e. $2.13 \mathrm{deg}^{2}$ ), to fluxes of $\sim 3.0 \times 10^{-15} \mathrm{erg} \mathrm{cm}^{-2} \mathrm{~s}^{-1}, \sim 1.5 \times 10^{-14} \mathrm{erg} \mathrm{cm}^{-2} \mathrm{~s}^{-1}$ and $\sim 2.0 \times 10^{-14} \mathrm{erg} \mathrm{cm}^{-2} \mathrm{~s}^{-1}$, respectively. As mentioned above, the fluxes of the input spectrum are converted into count-rates by assuming a single spectrum for all the sources. This could in principle bias the estimates of the sensitivity limits. In order to test the effect of a variation of the mean spectral index in the estimate of the sky coverage, we changed the spectral indices by $\Delta \Gamma= \pm 0.3$. In this way the estimate of the flux limit changed by $<2 \%$ in the soft band, while this variation was of the order of $9 \%$ and $4 \%$ in the $2-10 \mathrm{keV}$ and $5-10 \mathrm{keV}$ band. The sky coverage is thus almost insensitive to a change of the spectral shape in the $0.5-2 \mathrm{keV}$ and in the $5-10 \mathrm{keV}$ band. In the $2-10 \mathrm{keV}$ we estimated that a $9 \%$ uncertainty in the flux limit could introduce an overall uncertainty of $\sim 5 \%$ in the $\log N-\log S$. Such an uncertainty is however smaller than the typical uncertainty on the source counts.

In order to map the sensitivity across the field of view we produced sensitivity maps of the XMM-COSMOS survey in all the energy bands by reversing our source detection analysis. By using our estimated background maps and exposure maps we evaluated, according to the Poisson statistic, the minimum number of counts necessary to have a detection with det_ml $>10$. The number of counts have been evaluated in cells of $\overline{3} \times 3$ pixels and corrected for the fraction of the PSF falling out of the cell. The resulting count-limit maps have been divided by the exposure maps and converted into flux limit maps using the ECF.

As an example, the resulting $0.5-2 \mathrm{keV}$ band sensitivity map is plotted in colour scale in the right panel of Fig. 5. The map is in excellent agreement with the sky coverage plot obtained via Monte Carlo simulations. As one can notice, almost all the central area $\left(\sim 1.8 \mathrm{deg}^{2}\right)$ has a quite homogeneous flux limit $\sim 1.7 \times 10^{-15} \mathrm{erg} \mathrm{cm}^{-2} \mathrm{~s}^{-1}$. The northern central part of the field shows an area of $\sim 0.5 \mathrm{deg}^{2}$, having a flux limit of the order of $\sim 8 \times 10^{-16} \mathrm{erg} \mathrm{cm}^{-2} \mathrm{~s}^{-1}$. It is worth noting that the deepest part of the field is located in the northeastern part of the field and the flux limit of $\sim 5.0 \times 10^{-16} \mathrm{erg} \mathrm{cm}^{-2} \mathrm{~s}^{-1}$ in the sensitivity maps is in agreement with the predictions of the Monte Carlo simulations and the output of the source detection.

\section{4. $\log N-\log S$ relations}

Using the sky coverage we produced the cumulative $\log N-\log S$ relations in the three energy bands under investigation by using:

$N(>S)=\sum_{i=1}^{N_{S}} \frac{1}{\Omega_{i}} \operatorname{deg}^{-2}$

where $N(>S)$ is the total number of detected sources in the field with fluxes greater than $S$ and $\Omega_{i}$ is the sky coverage associated with the flux of the ith source. The variance of the source number counts is therefore defined as:

$\sigma_{i}^{2}=\sum_{i=1}^{N_{S}}\left(\frac{1}{\Omega_{i}}\right)^{2}$ 


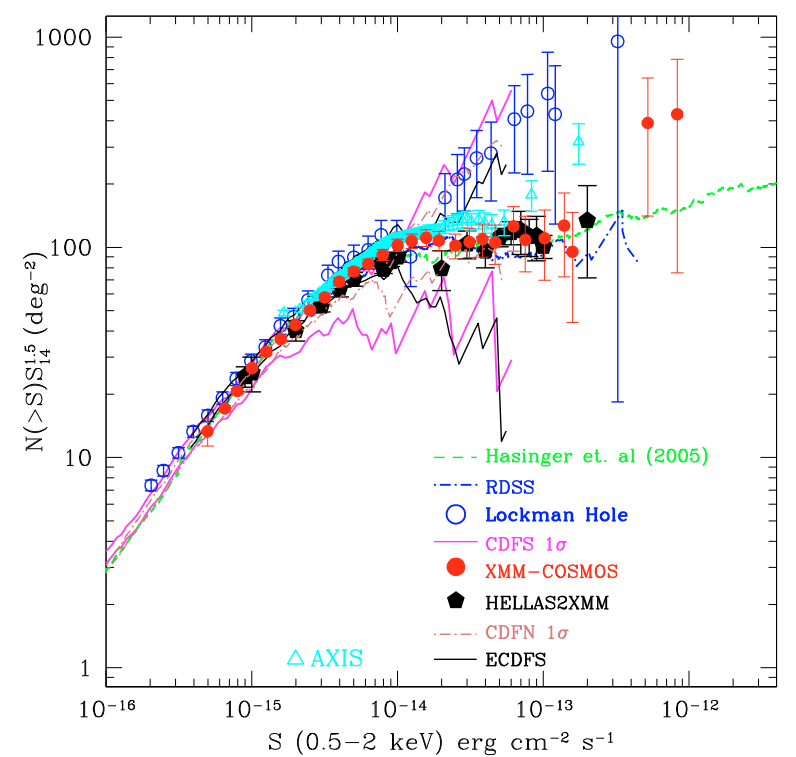

Fig. 6. The $0.5-2 \mathrm{keV} \log N-\log S$ of the XMM-COSMOS (red dots) sources compared with the ROSAT medium sensitivity survey (Hasinger et al. 1993, blue dot dashed line), combined ROSAT, XMMNewton, Chandra sources (Hasinger et al. 2005, green dashed line), the 2Ms CDFS ( $1 \sigma$ error tie, Luo et al. 2008, magenta continuous line), the 2 Ms CDFN ( $1 \sigma$ error tie, Bauer et al. 2004, pink dot - dashed line), the XMM-Newton Lockman hole (Brunner et al. 2008, blue circles), the AXIS (Carrera et al. 2007, cyan triangles), the HELLAS2XMM (Baldi et al. 2002, black pentagons) and the extended CDFS ( $1 \sigma$ error tie, Lehmer et al. 2005, black continuous line) surveys. The source number counts are plotted multiplied by $\left(S / 10^{14}\right)^{1.5}$ in order to highlight the deviations from the Euclidean behavior.

The cumulative number counts, normalized to the Euclidean slope (i.e. multiplied by $S^{1.5}$ ), are shown in Figs. 6-8, in the $0.5-2 \mathrm{keV}, 2-10 \mathrm{keV}$ and $5-10 \mathrm{keV}$ energy ranges, respectively. The $\log N-\log S$ relations are also presented in Table 4. From left to right: Flux, Number-counts and area in the $0.5-2 \mathrm{keV}$, 2-10 keV and 5-10 keV energy band, respectively.

In order to parametrize our relations, we performed a maximum likelihood fit to the unbinned differential counts. We assumed a broken power-law model for the $0.5-2 \mathrm{keV}$ and 2-10 keV bands:

$n(S)=\frac{\mathrm{d} N}{\mathrm{~d} s}= \begin{cases}A S^{-\alpha_{1}} & S>S_{\mathrm{b}} \\ B S^{-\alpha_{2}} & S \leq S_{\mathrm{b}}\end{cases}$

where $A=B S_{\mathrm{b}}^{\alpha_{1}-\alpha_{2}}$ is the normalization, $\alpha_{1}$ is the bright end slope, $\alpha_{2}$ the faint end slope, $S_{\mathrm{b}}$ the break flux, and $S$ the flux in units of $10^{-14} \mathrm{erg} \mathrm{cm}^{-2} \mathrm{~s}^{-1}$. Notice that, using the maximum likelihood method, the fit is not dependent on the data binning and therefore we are using the whole dataset. Moreover, the normalization $A$ is not a parameter of the fit, but is obtained by imposing the condition that the number of expected sources from the best fit model is equal to the total observed number.

In the $0.5-2 \mathrm{keV}$ energy band the best fit parameters are $\alpha_{1}=2.40 \pm 0.05, \alpha_{2}=1.60_{-0.10}^{+0.04}, S_{\mathrm{b}}=1.00_{-0.26}^{+0.21} \times$ $10^{-14} \mathrm{erg} \mathrm{cm}^{-2} \mathrm{~s}^{-1}$ and $A=141$. These values are consistent with those measured in Paper II while the normalization is lower than the value $(A=198)$ derived in Paper $\mathrm{II}^{6}$. However, with this fitting method the normalization is not a fit parameter and it is strongly dependent on the best fit values of the bright end

\footnotetext{
${ }^{6}$ Note that in Paper II we gave the normalization of the cumulative distributions.
}

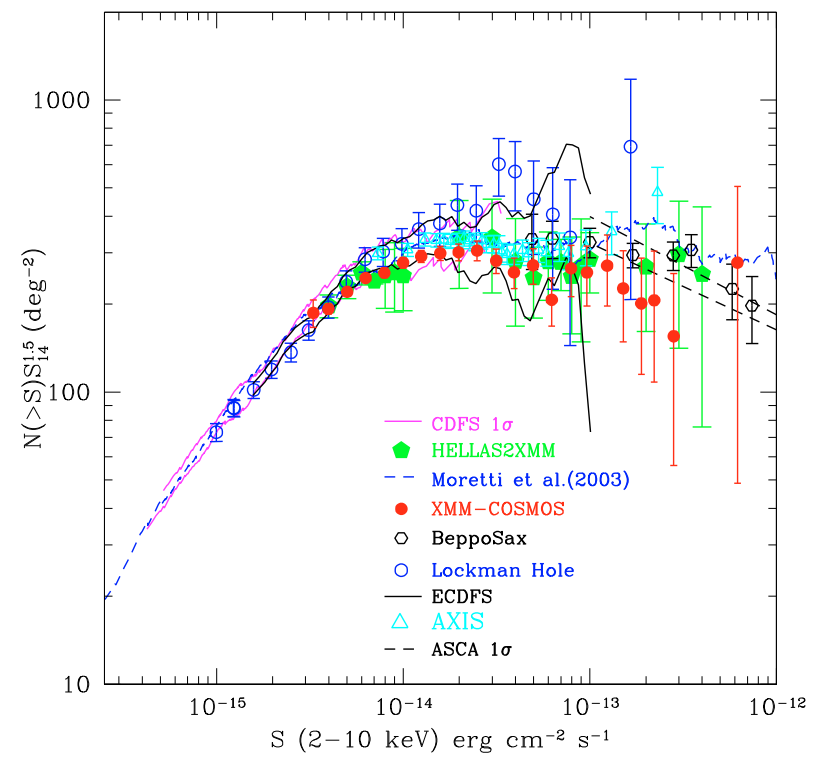

Fig. 7. The $2-10 \mathrm{keV} \log N-\log S$ of the XMM-COSMOS (red dots) sources compared with the combined Chandra, XMM-Newton and ASCA sources (Moretti et al. 2003, blue dashed line), the HELLAS BeppoSAX (Giommi et al. 2000, black hexagons) the 2 Ms CDFS ( $1 \sigma$ error tie, Luo et al. 2008, magenta continuous line), the HELLAS2XMM (Baldi et al. 2002, green pentagons), the AXIS (Carrera et al. 2007, cyan triangles), the extended CDFS ( $1 \sigma$ error tie, Lehmer et al. 2005, black continuous line) and the Lockman hole (Brunner et al. 2008, blue open circles) surveys. The black - dashed line are the $1 \sigma$ confidence contours of the best fit to the $\log N-\log S$ of the ASCA data (Cagnoni et al. 1998). The source number counts are plotted multiplied by $\left(S / 10^{14}\right)^{1.5}$ in order to highlight the deviations from the Euclidean behavior.

slope and on the cut-off flux. One can indeed notice that the best fit values of the $\alpha_{1}$ and $S_{\mathrm{b}}$ parameters are somewhat changed with respect to Paper II. The bright end slope varied from 2.6 in Paper II to 2.4 in the present work and the cut-off flux varied from $\sim 1.55 \times 10^{-14} \mathrm{erg} \mathrm{cm}^{-2} \mathrm{~s}^{-1}$ to $1.00 \times 10^{-14} \mathrm{erg} \mathrm{cm}^{-2} \mathrm{~s}^{-1}$. However, a comparison of the amplitude of the source surface density measured in Paper II with that measured here can be performed if we measure the model predicted source counts at fluxes fainter than the knee. If we take $2 \times 10^{-15} \mathrm{erg} \mathrm{cm}^{-2} \mathrm{~s}^{-1}$ as a reference flux, in Paper II we had a source surface density of $478 \mathrm{deg}^{-2}$ while here we measure $479 \mathrm{deg}^{-2}$. We can therefore conclude that the $0.5-2 \mathrm{keV} \log N-\log S$ obtained in Paper II and in this work are in good agreement.

In the $2-10 \mathrm{keV}$ band the best fit parameters are $\alpha_{1}=2.46 \pm$ $0.08, \alpha_{2}=1.55 \pm 0.18, S_{\mathrm{b}}=1.05 \pm 0.1610^{-14} \mathrm{erg} \mathrm{cm}^{-2} \mathrm{~s}^{-1}$ and $A=413$. Since the best fit parameters are similar to those of Paper II, we can directly compare the normalizations of the $\log N-\log S$. The normalization derived in this work is $10 \%$ higher than that measured in Paper II. This effect is partly due to the sources missing in the $2-4.5 \mathrm{keV}$ band and detected in the 2-8 keV which were not considered in the analysis of Paper II. Moreover, extrapolation of the $2-4.5 \mathrm{keV}$ count-rate into the broader $2-10 \mathrm{keV}$ band is more affected by uncertainties on the true source spectral slope and provides wrong count-rate estimates especially for the most absorbed sources.

In the $5-10 \mathrm{keV}$ energy band we did not find any significant break in the slope. We therefore fitted the data using a single power-law in the form of $n(S)=A S^{-\alpha_{1}}$ and obtained $\alpha_{1}=2.38 \pm$ 0.05 and $A=130$.

In the $5-10 \mathrm{keV}$ both the normalization and the slope are consistent within $1 \sigma$ with the values obtained in Paper II. 
Table 4. Source number counts.

\begin{tabular}{|c|c|c|c|c|c|c|}
\hline$\overline{\log (S)}$ & $N(>S)$ & Area & $\overline{N(>S)}$ & Area & $N(>S)$ & Area \\
\hline $\mathrm{erg} \mathrm{cm}^{-2} \mathrm{~s}^{-1}$ & $\begin{array}{c}\mathrm{deg}^{-2} \\
0.5-2 \mathrm{keV}\end{array}$ & $\operatorname{deg}^{2}$ & $\begin{array}{c}\mathrm{deg}^{-2} \\
2-10 \mathrm{keV}\end{array}$ & $\operatorname{deg}^{2}$ & $\begin{array}{c}\mathrm{deg}^{-2} \\
5-10 \mathrm{keV}\end{array}$ & $\operatorname{deg}^{2}$ \\
\hline-12.8 & $1.51 \pm 0.81$ & 2.13 & $3.86 \pm 1.33$ & 2.13 & $1.04 \pm 0.66$ & 2.13 \\
\hline-12.9 & $2.45 \pm 1.05$ & 2.13 & $6.20 \pm 1.69$ & 2.13 & $1.98 \pm 0.94$ & 2.13 \\
\hline-13.0 & $3.39 \pm 1.24$ & 2.13 & $8.55 \pm 1.99$ & 2.13 & $2.92 \pm 1.15$ & 2.13 \\
\hline-13.1 & $5.26 \pm 1.56$ & 2.13 & $11.84 \pm 2.35$ & 2.13 & $4.79 \pm 1.48$ & 2.13 \\
\hline-13.2 & $8.08 \pm 1.94$ & 2.13 & $13.25 \pm 2.48$ & 2.13 & $7.14 \pm 1.82$ & 2.13 \\
\hline-13.3 & $10.43 \pm 2.20$ & 2.13 & $24.51 \pm 3.39$ & 2.13 & $8.55 \pm 1.99$ & 2.13 \\
\hline-13.4 & $14.65 \pm 2.61$ & 2.13 & $32.96 \pm 3.93$ & 2.13 & $11.84 \pm 2.35$ & 2.13 \\
\hline-13.5 & $19.35 \pm 3.01$ & 2.13 & $50.33 \pm 4.86$ & 2.13 & $17.94 \pm 2.89$ & 2.13 \\
\hline-13.6 & $25.92 \pm 3.48$ & 2.13 & $77.56 \pm 6.03$ & 2.13 & $24.98 \pm 3.42$ & 2.13 \\
\hline-13.7 & $40.48 \pm 4.35$ & 2.13 & $108.07 \pm 7.12$ & 2.13 & $39.07 \pm 4.28$ & 2.13 \\
\hline-13.8 & $56.44 \pm 5.14$ & 2.13 & $150.33 \pm 8.40$ & 2.13 & $55.57 \pm 5.11$ & 2.10 \\
\hline-13.9 & $76.63 \pm 5.99$ & 2.13 & $208.52 \pm 9.90$ & 2.09 & $84.72 \pm 6.36$ & 1.82 \\
\hline-14.0 & $102.45 \pm 6.93$ & 2.13 & $277.20 \pm 11.48$ & 1.98 & $123.75 \pm 8.26$ & 0.95 \\
\hline-14.1 & $131.09 \pm 7.84$ & 2.13 & $361.50 \pm 13.31$ & 1.77 & $166.26 \pm 13.33$ & 0.23 \\
\hline-14.2 & $166.77 \pm 8.85$ & 2.13 & $491.38 \pm 16.27$ & 1.33 & $212.88 \pm 43.57$ & 0.02 \\
\hline-14.3 & $217.00 \pm 10.09$ & 2.13 & $620.89 \pm 19.91$ & 0.67 & & \\
\hline-14.4 & $273.34 \pm 11.33$ & 2.13 & $766.57 \pm 29.63$ & 0.14 & & \\
\hline-14.5 & $324.98 \pm 12.35$ & 2.13 & $984.00 \pm 106.76$ & 0.01 & & \\
\hline-14.6 & $398.23 \pm 13.67$ & 2.13 & & & & \\
\hline-14.7 & $480.54 \pm 15.04$ & 2.06 & & & & \\
\hline-14.8 & $581.69 \pm 16.68$ & 1.80 & & & & \\
\hline-14.9 & $713.44 \pm 19.05$ & 1.37 & & & & \\
\hline-15.0 & $842.39 \pm 21.69$ & 1.00 & & & & \\
\hline-15.1 & $930.12 \pm 24.30$ & 0.44 & & & & \\
\hline-15.2 & $1027.83 \pm 35.07$ & 0.08 & & & & \\
\hline-15.3 & $1201.41 \pm 177.09$ & 0.01 & & & & \\
\hline
\end{tabular}

\subsection{Comparison with previous surveys}

In Figs. 6-8 we compare our $\log N-\log S$ with the results of previous surveys. A visual inspection of the data shows that the XMM-COSMOS source counts are in general agreement, within $1 \sigma$, with all the previous measurements. In the $0.5-2 \mathrm{keV}$ band source counts of all the surveys agree with our measurements, with the only exception of the bright end of the Lockman Hole $\log N-\log S$. The reason for such a discrepancy is that the location of the Lockman Hole survey was chosen on purpose near a concentration of bright sources to improve the accuracy of the ROSAT star tracker in order to achieve a better astrometry (Hasinger, private communication). This had the result of artificially increasing the source counts at the bright end of the relation. The comparison with other surveys is consistent with the error bars and with the counts in cell fluctuations predicted in Paper II. We also compared our results with the recent work of Mateos et al. (2008) who performed a detailed analysis of the $\log N-\log S$ of X-ray sources detected in 1129 XMMNewton archival observations. By comparing the data of Table 4 with those shown in Table 3 of Mateos et al. (2008) we found $1 \sigma$ agreement in almost all the data bins.

In Paper II we showed that the fluctuations of the source counts are proportional to the actual number of sources in the field and to the amplitude of the angular auto-correlation function of the X-ray sources. Therefore, assuming a universal shape of the autocorrelation function, we expect that the surveys showing the largest deviations from the mean value of the source density are the pencil beam surveys (i.e. area $<0.2 \mathrm{deg}^{2}$ ) at their bright end. Moreover, with XMM-COSMOS , fluctuations introduced in previous shallow surveys by low counting statistics and by random sampling of a few large structures in pencil beam surveys are largely suppressed. With the same formulas used in Paper II, we estimate the effect of the cosmic variance to be $<5 \%$ on the normalization of the XMM-COSMOS $\log N-\log S$ and that the new data do not change the results shown in Paper II.

Also in the $2-10 \mathrm{keV}$ energy bands we do not note any significant deviation from previous works with the exception only of the Lockman Hole and the two faintest bins of the AXIS counts. Also in this band our data are in good agreement with the results of Mateos et al. (2008).

Figure 8 suggests that the fluctuations of the source counts in the 5-10 keV band are much larger than in the other bands. This is due to the fact that as discussed above and in Paper II, when we deal with low source surface density, the impact of the sample variance becomes significantly high. However in this band our data are statistically consistent with most of the data from other surveys. Also in this energy band, the deviation of the COSMOS data from those of the Lockman Hole is due the higher number of bright sources in that particular field. Our source counts are $10-15 \%$ higher than those of the ELAIS-S1 survey (Puccetti et al. 2006). As an example, using Eq. (10) of Paper II, we determine that at the faint end of the $5-10 \mathrm{keV}$ band, fluctuations due to the cosmic variance are of the order of the 20-40 percent, depending on the survey size. At the bright end large deviations of more than a factor of two are still allowed by the sample variance. This is also visible in Fig. 8 where at the bright end the Beppo-SAX counts exceed the XMM-COSMOS counts by about a factor of two though remaining statistically consistent with each other.

Kim et al. (2007) reported the results of a broken power law fit to the $\log N-\log S$ from different surveys available in the literature. They also reported measurements of the CHAMP survey which is a compilation of Chandra archival data for a total 


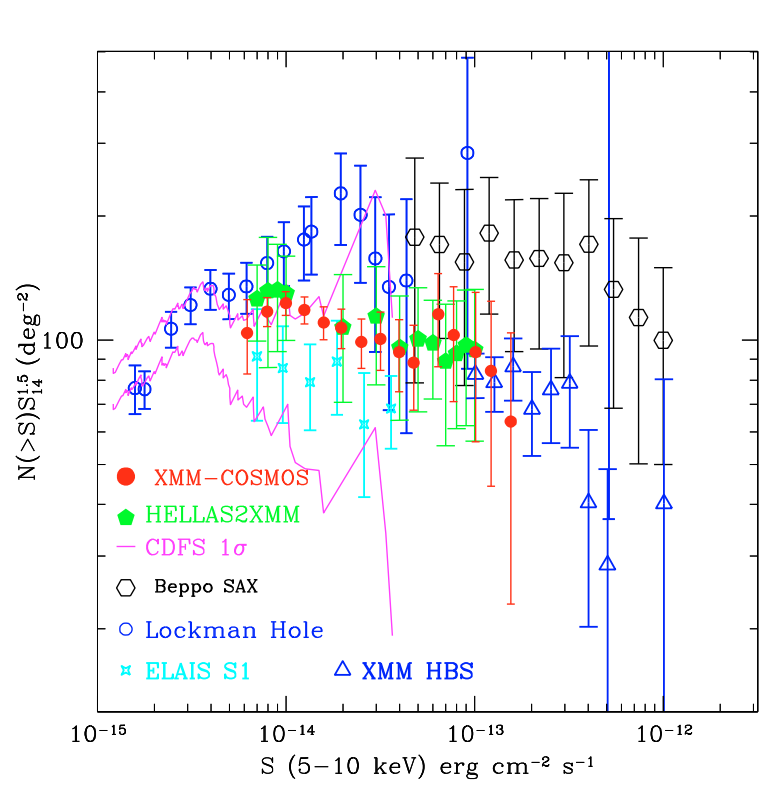

Fig. 8. The 5-10 keV $\log N-\log S$ of the XMM-COSMOS (red dots) sources compared with the HELLAS2XMM (Baldi et al. 2002, green pentagons), the 1 Ms CDFS ( $1 \sigma$ error tie, Rosati et al. 2002, magenta continuous line), the HELLAS-BeppoSAX (Fiore et al. 2001, black hexagons), the ELAIS S1 (Puccetti et al. 2006, blue stars), the XMM-HBS (Della Ceca et al. 2004, blue triangles) and the Lockman Hole (Brunner et al. 2008, blue open circles). surveys. The source number counts are plotted multiplied by $\left(S / 10^{14}\right)^{1.5}$ in order to highlight deviations from the Euclidean behavior.

sky coverage of $9.6 \mathrm{deg}^{2}$, with a depth about one order of magnitude fainter than XMM-COSMOS. On average the bright end slopes are consistent with a Euclidean rise in all the surveys. The faint end slopes are of the order of $\alpha_{2} \sim 1.5-1.6$ in the 0.5$2 \mathrm{keV}$ band and span from $\alpha_{2} \sim 1.3$ to $\alpha_{2} \sim 2.0$ with a mean of $\alpha_{2} \sim 1.6-1.7$. A larger spread is reported for the cut off fluxes. Although the spread in this parameter is quite large, our data are consistent with the average values reported in the literature for this parameter.

\subsection{Extragalactic $X$-ray source number counts and comparison with models}

We used our $\log N-\log S$ relations to test the most recent extragalactic XRB synthesis models. In order to compare our data with the XRB model, we estimate the fraction of sources classified as stars by Brusa et al. (2009). In the 0.5-2 keV band we identified 74/1621 (i.e. 4.5\%) sources classified as stars, while these are $17 / 1111$ (i.e. $\sim 1.5 \%$ ) and $3 / 251$ (i.e. $\sim 1.1 \%$ ) in the 2-10 keV and 5-10 keV bands, respectively. In Fig. 9 we plot the normalized distributions of the fluxes of stars and extragalactic sources in the $0.5-2 \mathrm{keV}$ band. Since the two distributions are similar we can conclude that stars in the XMM-COSMOS flux range affect the $0.5-2 \mathrm{keV} \log N-\log S$ only by increasing the extragalactic source counts by $\sim 5 \%$. Mateos et al. (2008) measured a flux dependent fraction of stars, with higher fractions than ours at bright fluxes where XMM-COSMOS is undersampled. By excluding the source classified as stars, we derived the $\log N-\log S$ relations for extragalactic sources only.

In the upper panel of Fig. 10 we plot the ratio of the XMM-COSMOS $\log N-\log S$ relations to the predictions of the XRB population synthesis model of Gilli et al. (2007, hereafter model I), while in the bottom panel we plot for comparison

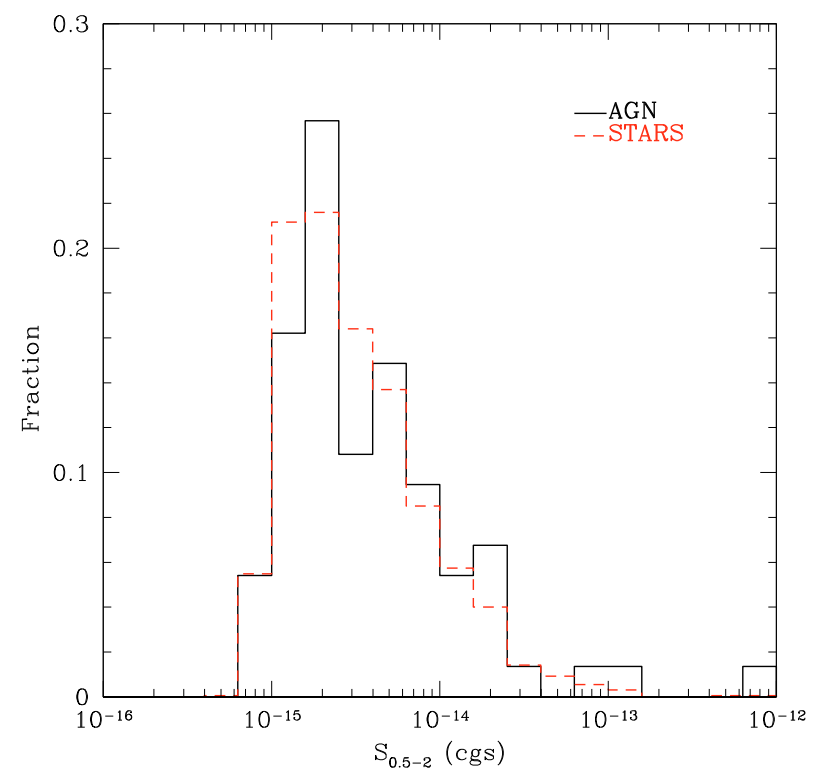

Fig. 9. The 0.5-2 keV flux distribution of sources classified as AGN or extragalactic (black) and stars (red).

the ratio of the data to the model of Treister \& Urry (2006) (hereafter model II). In both models the XRB spectrum is dominated by obscured AGN which outnumber unobscured ones by a factor 3-4 at low X-ray luminosities $\left(\log L_{X}<44\right)$. The cosmological evolution is similar and parametrized using the most recent determinations of the AGN luminosity function (Ueda et al. 2003; La Franca et al. 2005; Hasinger et al. 2005). In both models the obscured fraction decreases towards high luminosity. The luminosity dependence is stronger in Treister et al. (2006) who also allow the obscured fraction to increase at high redshifts. The absorption distribution is peaked around $\log N_{\mathrm{H}} \sim 23.5$ in Gilli et al. (2007), while it remains rather flat above $\log N_{\mathrm{H}} \sim 22$ in Treister et al. (2006). They also differ in the adopted XRB intensity around the $30 \mathrm{keV}$ peak. The Gilli et al. (2007) model is tuned to fit the HEAO-1 level, consistent, within $10 \%$, with recent BeppoSAX (Frontera et al. 2007) and Swift BAT (Ajello et al. 2008) measurements, while Treister et al. renormalize the HEAO-1 intensity upward by a factor 1.4 to better match the extrapolation of lower energy $(<10 \mathrm{keV})$ data (i.e. De Luca \& Molendi 2004). Moreover, for this paper we adopt a modified version of the Gilli et al. (2007) model $^{7}$ which takes into account the decline of the space density of AGN at $z>3$ discussed by Brusa et al. (2008). In order to test the models over a wider range of fluxes we also plotted the data of the CDFN (Bauer et al. 2004) and CDFS (Luo et al. 2008) surveys. By restricting our analysis to fluxes larger than $10^{-16} \mathrm{erg} \mathrm{cm}^{-2} \mathrm{~s}^{-1}$, the contribution of normal galaxy counts is negligible (Ranalli et al. 2003). The results of this comparison can be summarized as follows:

- in the 5-10 keV energy band, both models reproduce well the XMM-COSMOS $\log N-\log S$, while the CDFS counts show a systematically different slope from that of the predicted relation. However, because of the small effective area of Chandra above $5 \mathrm{keV}$ (i.e. $\sim 200 \mathrm{~cm}^{2}$ at $6.4 \mathrm{keV}$ ), the 5$10 \mathrm{keV} C D F S \log N-\log S$ may suffer from significant systematic uncertainties.

7 The predictions of the model can be retrieved on line at http://
wWw. oabo.inaf.it/ gilli/counts.html using the POMPA
COUNTS software (POrtable Multi Purpose Application for the AGN COUNTS). 

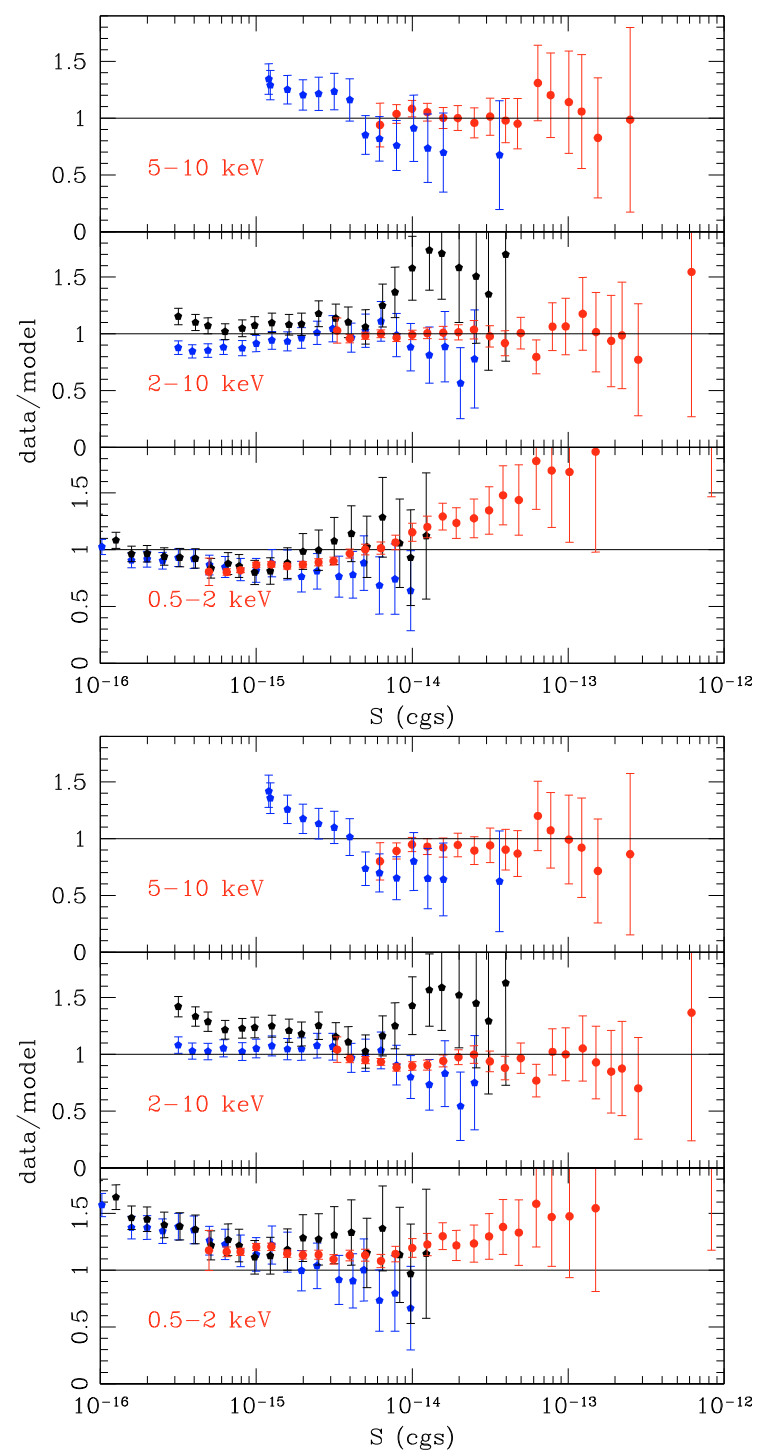

Fig. 10. Upper panel: the ratio between the Gilli et al. (2007) model $\log N-\log S$ relations to the observed source counts in XMMCOSMOS and in the Chandra deep fields (Rosati et al. 2002; Bauer et al. 2004). From bottom to top in the $0.5-2 \mathrm{keV}, 2-10 \mathrm{keV}$ and 5-10 keV energy bands. The XMM-COSMOS datapoints are plotted in red, while in black and blue we plot the CDFN and CDFS, respectively. Bottom panel: same as Upper panel but using the Treister \& Urry (2006) XRB model.

- in the 2-10 keV energy band, the models reproduce quite accurately the XMM-COSMOS data, although model II slightly (i.e. $\sim 10 \%$ ) overpredicts the XMMCOSMOS counts. The CDFN counts show a systematically higher normalization than those of the models (up to $40 \%$ at faint fluxes) and of the COSMOS and CDFS data.

- in the $0.5-2 \mathrm{keV}$ band both models show significant deviations from both data sets. Source counts estimated from model I show a systematically steeper slope than the data. On average, model I deviates from the observations by about $10-15 \%$ in the flux range $10^{-16} \mathrm{erg} \mathrm{cm}^{-2} \mathrm{~s}^{-1}-10^{-13}$ $\operatorname{erg} \mathrm{cm}^{-2} \mathrm{~s}^{-1}$. Model II, on the other hand, systematically underestimates the source counts while a visual inspection shows a good agreement with the observed slopes. In this case deviations between the data and the model are of the order of $20 \%$ at the XMM-COSMOS fluxes, while at fainter fluxes the deviations are larger and of the order of $30 \%-40 \%$. Both models underestimate the observed counts by $\sim 30 \%$ at fluxes greater than $\sim 10^{-14} \mathrm{erg} \mathrm{cm}^{-2} \mathrm{~s}^{-1}$

In summary, the hard X-ray observations are very well reproduced by both models with an accuracy of $\sim 10 \%$. In the soft band the agreement between the predicted and the observed relations is not as good as in the harder energy bands ${ }^{8}$. The level of the discrepancy, however, is small $(\sim 20 \%)$ and such that it can be easily accommodated by slight variations of the XRB model parameters. This band is in fact more sensitive to the effect of absorption and therefore a fine tuning of absorption in AGN is required in the models. Moreover, this band contains a larger fraction of high- $z$ objects (Brusa et al. 2009). Therefore, the fact that the two models assume a somewhat different absorption evolution and XRB spectrum can, in the first instance, explain the different source count predictions. We can conclude that at the flux limits of the XMM-COSMOS survey, XRB synthesis models can reproduce the observations with a precision of $10 \%-20 \%$.

\section{X-ray colours of the X-ray sources}

The X-ray colours or hardness ratios are defined as

$H R_{1}=\frac{B_{2}-B_{1}}{B_{2}+B_{1}}$ and $H R_{2}=\frac{B_{3}-B_{2}}{B_{3}+B_{2}}$

where $B_{1}, B_{2}$, and $B_{3}$ refer to the vignetting-corrected count rates in the $0.5-2 \mathrm{keV}, 2-10 \mathrm{keV}$ and $5-10 \mathrm{keV}$ bands, respectively. By construction, both HR1 and HR2 can assume values between -1 and 1.

Figure 11 displays the HR1-HR2 plot of 212 sources for which the $1 \sigma$ error on both HR 1 and HR2 is $<0.25$ and for which a high quality optical spectrum is available. The plot also contains a grid of the expected values of HR1 and HR2 for different spectral models. In particular we considered a simple power law model with a spectral index in the interval $\Gamma=0 \div 3$ and with a column density $\log \left(N_{\mathrm{H}}\right)=0 \div 23 \mathrm{~cm}^{-2}$.

In Brusa et al. (2009), and Trump et al. (2008), extragalactic sources are classified into 4 main categories:

- type I AGN, if the optical spectrum shows evidence of broad $\left(F W H M>2000 \mathrm{~km} \mathrm{~s}^{-1}\right)$ emission lines;

- type II AGN, if the optical spectrum shows evidence of narrow, high-ionization emission lines and/or AGN diagnostic diagrams;

- emission line galaxy, if the optical spectrum is dominated by a galaxy continuum plus emission lines but without secure AGN indicators;

- absorption line galaxy if the optical spectrum is dominated by a galaxy continuum plus absorption lines.

Details of the optical classification of X-ray sources are extensively discussed in Brusa et al. (2009), therefore we limit our analysis to the X-ray properties of these sources. 140/212 are classified as type I, 32/212 as type II, 30/212 as emission line galaxies and 10/212 as absorption line galaxies. Note that with the exception only of 7 objects, all the sources have an estimated 2-10 keV X-ray luminosity $\log \left(L_{\mathrm{X}}\right)>42 \mathrm{erg} / \mathrm{s}$, with most of them having $\log \left(L_{X}\right)>43 \mathrm{erg} / \mathrm{s}$ (see Fig. 12). The adopted cuts

\footnotetext{
8 Note that the inclusion of a decline in the space density of AGN at high- $z$ in model I affects mostly the $0.5-2 \mathrm{keV}$ energy bands. In the harder bands the predicted number counts are comparable with or without a high- $z$ space density decline.
} 


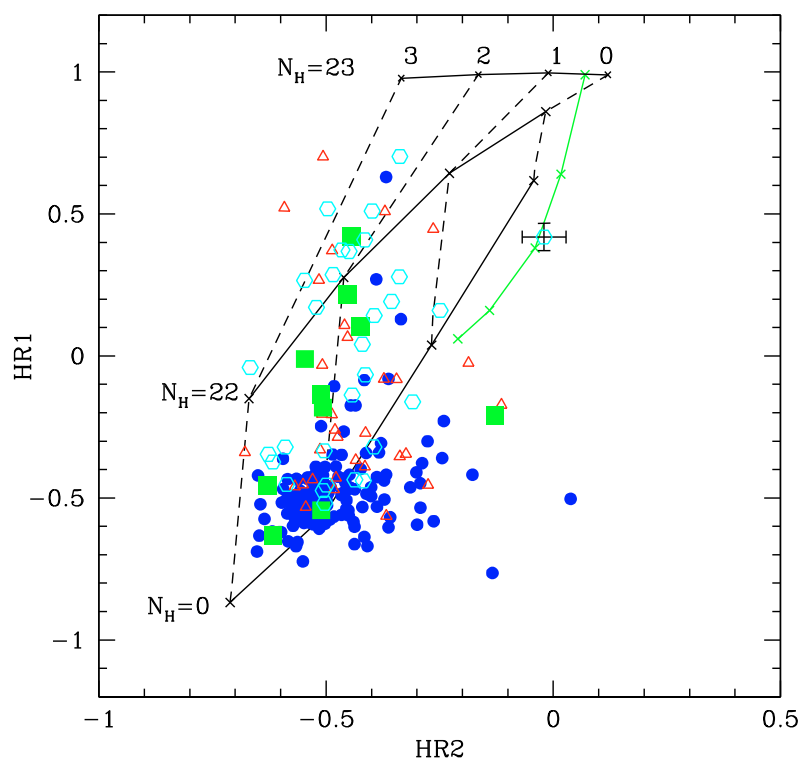

Fig. 11. X-ray colour-colour diagram in the XMM-COSMOS survey. Colours are defined in the text. XID \#2608 has been plotted with its error which also represents the typical amplitude of the uncertainties in the plot. The grid represents the places in the HR1-HR2 plane of sources with single power-law spectra with $\Gamma=0 \div 3$ with absorption with a column density $\log \left(N_{\mathrm{H}}\right)=0 \div 23 \mathrm{~cm}^{-2}$. The green line marks the region occupied by candidate Compton thick-AGN [i.e. $\log \left(N_{\mathrm{H}}>24\right) \mathrm{cm}^{-2}$ ] and the marks on top of it represent 1\%,3\% 10\% and 30\% level of leaking flux, from top to bottom. We represent type I AGN, type II AGN, emission line and absorption line galaxies as blue filled circles, red empty triangles, cyan empty exagons, green filled squares, respectively.

on the errors on the HR preferentially select unabsorbed to moderate absorbed AGN, biasing the sample against normal galaxies, starforming galaxies and the most obscured AGN.

type I AGN (blue filled circles) cluster in a region around $\mathrm{HR} 1=-0.5$ and HR2 $=-0.5$ with a relatively small dispersion, corresponding to a typical X-ray spectrum dominated by a power-law continuum with very low absorption. Only a few type I sources have X-ray colours typical of type II sources (i.e. HR $>-0.1$ which corresponds to $N_{H}>10^{22} \mathrm{~cm}^{-2}$ ). This fraction $(\sim 2 \%)$ is consistent with the results from X-ray spectral analysis on a subsample of XMM-COSMOS sources (Mainieri et al. 2007) but at variance with previous works on the fraction of $\mathrm{X}$-ray absorbed type I AGN at comparable X-ray luminosity (see e.g. Brusa et al. 2003; Perola et al. 2004; Page et al. 2004) which reported values as large as $10 \%$. However such a low fraction may be a consequence of the selection effect mentioned above.

On the other hand, type II AGN (red empty triangles) fill most of the HR1-range, corresponding to observed frame absorption up to $10^{23} \mathrm{~cm}^{2}$. The fraction of type II AGN with X-ray colours typical of type I AGN (HR1<-0.3) is $\sim 30 \%$. This is consistent with the fraction of X-ray unobscured type II AGN reported in Mainieri et al. (2007).

An interesting source is XID = \#2608 which has been classified as a Compton-Thick AGN by Mainieri et al. (2007) and Hasinger et al. (2007) but its optical spectrum is that of an emission line galaxy. In Hasinger et al. (2007) a small number of sources (including XID = \#2608) was found to have hardness ratios that could be interpreted as being due to heavily absorbed (possibly Compton thick) high energy spectra with some fraction of leaking unabsorbed soft flux. The solid green line in Fig. 11 represents the expected tracks occupied by leaking Compton

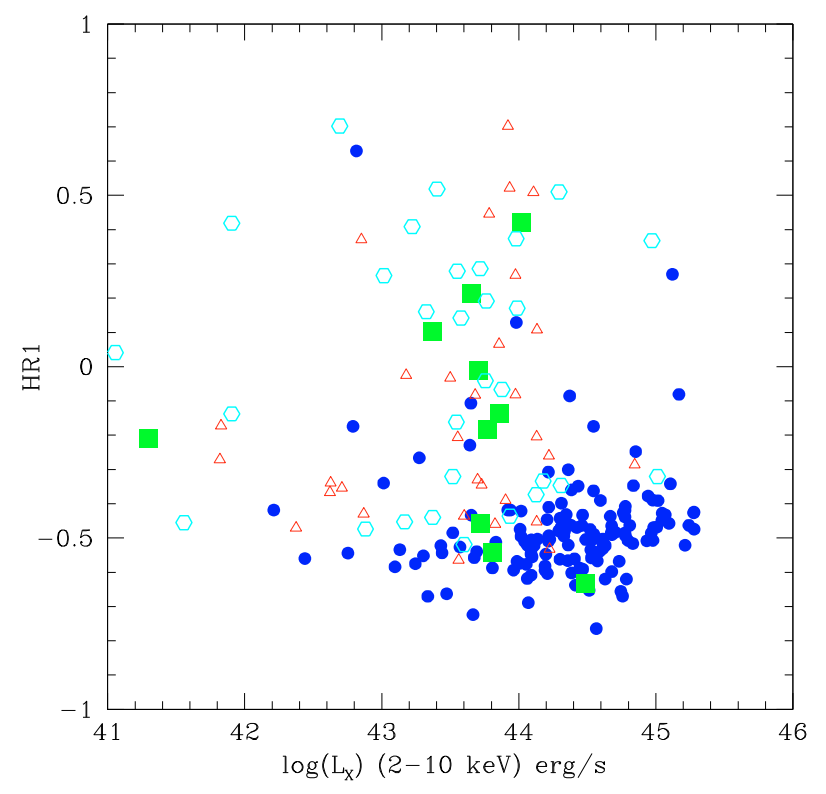

Fig. 12. The 2-10 keV X-ray luminosity vs. HR1 for the sources fullfilling the HR error selection. We represent type I AGN, type II AGN, emission line and absorption line galaxies as blue filled circles, red empty triangles, cyan empty exagons, green filled squares, respectively.

thick sources in the HR1-HR2 plane $^{9}$ at $z=0$. The line has been computed with a pure reflection model with a fraction of $1 \%, 3 \%, 10 \%$ and $30 \%$ (from top to bottom) of the flux from the central source leaking out.

In particular the source ID $=\# 2608$ shows X-ray colours typical of a spectrum dominated by a pure reflection component with $\sim 3 \%$ of the original flux leaking out. Another source, XID = \#131, shows X-ray colours consistent with Comptonthick AGN with a small fraction of leaking flux. We note that a $1 \%$ fraction of Compton-thick AGN is consistent with the predictions of XRB models at the flux limit of this subsample (i.e. 2-10 keV flux $>10^{-14} \mathrm{erg} \mathrm{cm}^{-2} \mathrm{~s}^{-1}$ ) and with the source counts of Compton-thick objects measured in a collection of surveys by Brunner et al. (2008).

The objects classified as emission and absorption line galaxies are spread over the entire luminosity-hardness ratio plane (see Fig. 12) and their nature can be explained as a mixture of star forming galaxies, type II AGN and XBONGs (see e.g. Comastri et al. 2002; Caccianiga et al. 2007; Civano et al. 2007; Cocchia et al. 2007). A more detailed analysis of their multiwavelength properties will be the subject of a forthcoming publication.

\section{Summary}

In this paper we presented a pointlike source catalogue in the XMM-COSMOS survey. The survey covers an area of $2.13 \mathrm{deg}^{2}$ in the equatorial sky. The field has been observed with 55 XMMNewton pointings for a total exposure time of $\sim 1.5 \mathrm{Ms}$. We achieved an almost uniform exposure of $\sim 40 \mathrm{ks}$ on the field.

We detected a total number of 1621, 1111 and 251 sources in the $0.5-2 \mathrm{keV}, 2-10 \mathrm{keV}$ and 5-10 keV energy band,

9 The position in this HR1-HR2 plane of the track of leaking Compton
thick objects is different from that shown in Hasinger et al. (2007) be-
cause of the difference in the energy range of the hard band (i.e. $2-8 \mathrm{keV}$ here, 2-4.5 keV in Hasinger et al. 2007). 
respectively, for a total of 1887 independent sources detected with det_ml $>10$ in at least one band. The survey has a limiting flux of $\sim 1.7 \times 10^{-15} \mathrm{erg} \mathrm{cm}^{-2} \mathrm{~s}^{-1}, \sim 9.3 \times 10^{-15} \mathrm{erg} \mathrm{cm}^{-2} \mathrm{~s}^{-1}$ and $\sim 1.3 \times 10^{-14} \mathrm{erg} \mathrm{cm}^{-2} \mathrm{~s}^{-1}$ in the $0.5-2 \mathrm{keV}, 2-10 \mathrm{keV}$ and 5$10 \mathrm{keV}$ energy band, over $90 \%$ of the area.

Together with the source catalogue we derived $\log N-\log S$ relations with high statistics in the flux interval sampled by the survey. The $\log N-\log S$ relations are in good agreement with most of the X-ray surveys published in the literature. We compared our source counts with the most recent XRB population synthesis models (Gilli et al. 2007; Treister \& Urry 2006) and found that they agree within $10 \%$ with our data in the $5-10 \mathrm{keV}$ and $2-10 \mathrm{keV}$ energy bands. In the $0.5-2 \mathrm{keV}$ band both models deviate from the XMM-COSMOS data by about 10\%-30\% suggesting, that further improvements in the modeling are required. We isolated a subsample of X-ray bright sources for which optical spectroscopy is available. About $65 \%$ of them have optical and X-ray properties typical of type I AGN and $\sim 15 \%$ of type II AGN. In the subsample of sources with a good optical spectrum and good counting statistics, the number of candidate Compton thick (1-2) AGN is fully consistent with the expectations of XRB population synthesis models. By combining $X$-ray colours and optical spectroscopy we found that $20 \%$ of the sources do not show, in the optical band, evident signatures of AGN activity although their X-ray luminosities are typical of AGN. Additonally, we consider XMM-COSMOS as a pathfinder for the eROSITA (Predehl et al. 2006) X-ray telescope which will be launched in 2012 and that will perform an all sky survey with sensitivities comparable to those presented here.

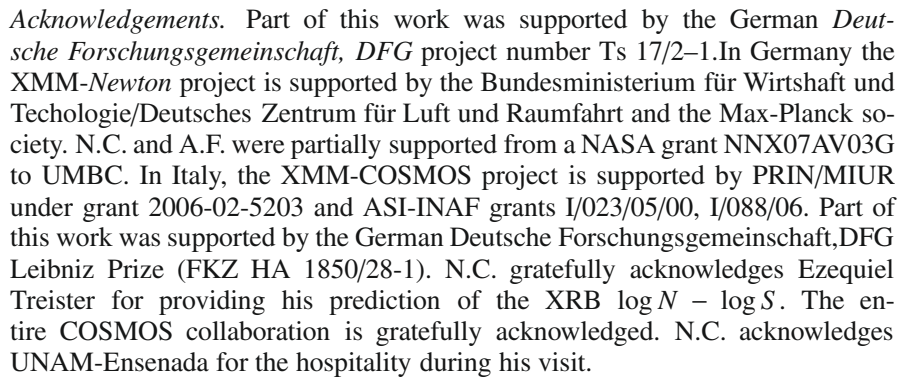

\section{References}

Ajello, M., et al. 2008, [arXiv: 0808 . 3377]

Baldi, A., Molendi, S., Comastri, A., et al. 2002, ApJ, 564, 190

Bauer, F. E., Alexander, D. M., Brandt, W. N., et al. 2004, AJ, 128, 2048

Branchesi, M., Gioia, I. M., Fanti, C., Fanti, R., \& Cappelluti, N. 2007, A\&A, 462, 449

Brunner, H., Cappelluti, N., Hasinger, G., et al. 2008, A\&A, 479, 283

Brandt, W. N., \& Hasinger, G. 2005, ARA\&A, 43, 827

Brusa, M., Comastri, A., Gilli, R., et al. 2008, [arXiv: 0809.2513]

Brusa, M., et al. 2009, A\&A, in preparation

Caccianiga, A., Severgnini, P., Della Ceca, R., et al. 2007, A\&A, 470, 557

Cagnoni, I., della Ceca, R., \& Maccacaro, T. 1998, ApJ, 493, 54

Cappelluti, N., Cappi, M., Dadina, M., et al. 2005, A\&A, 430, 39

Cappelluti, N., Hasinger, G., Brusa, M., et al. 2007a, ApJS, 172, 341

Cappelluti, N., Böhringer, H., Schuecker, P., et al. 2007b, A\&A, 465, 35

Cappi, M., Mazzotta, P., Elvis, M., et al. 2001, ApJ, 548, 624

Carrera, F. J., Ebrero, J., Mateos, S., et al. 2007, A\&A, 469, 27

Cavaliere, A., \& Fusco-Femiano, R. 1976, A\&A, 49, 137

Civano, F., Mignoli, M., Comastri, A., et al. 2007, A\&A, 476, 1223

Cocchia, F., Fiore, F., Vignali, C., et al. 2007, A\&A, 466, 31

Comastri, A., Mignoli, M., Ciliegi, P., et al. 2002, ApJ, 571, 771

Della Ceca, R., Maccacaro, T., Caccianiga, A., et al. 2004, A\&A, 428, 383

De Luca, A., \& Molendi, S. 2004, A\&A, 419, 837
Elvis, M., et al. 2009, [arXiv:0903.2062]

Finoguenov, A., Guzzo, L., Hasinger, G., et al. 2007, ApJS, 172, 182

Finoguenov, A., et al. 2008, ApJ, in preparation

Fiore, F., Giommi, P., Vignali, C., et al. 2001, MNRAS, 327, 771

Fiore, F., Brusa, M., Cocchia, F., et al. 2003, A\&A, 409, 79

Frontera, F., Orlandini, M., Landi, R. et al. 2007, ApJ, 666, 86

Gilli, R., Comastri, A., \& Hasinger, G. 2007, A\&A, 463, 79

Giommi, P., Perri, M., \& Fiore, F. 2000, A\&A, 362, 799

Hasinger, G., Burg, R., Giacconi, R., et al. 1993, A\&A, 275, 1

Hasinger, G., Miyaji, T., \& Schmidt, M. 2005, A\&A, 441, 417

Hasinger, G., Cappelluti, N., Brunner, H., et al. 2007, ApJS, 172, 29

Hathaway, D. H., Wilson, R. M., \& Reichmann, E. J. 1999, J. Geophys. Res., 104,22375

Kenter, A., Murray, S. S., Forman, W. R., et al. 2005, ApJS, 161, 9

Kim, M., Kim, Dong-Woo, Wilkes, B. J., et al. 2007, ApJS, 169, 401

Kocevski, D. D., Lubin, L. M., Gal, R., et al. 2008, [arXiv: 0804 . 1955]

La Franca, F., Fiore, F., Comastri, A., et al. 2005, ApJ, 635, 864

Lehmer, B. D., Brandt, W. N., Alexander, D. M., et al. 2005, ApJS, 161, 21

Luo, B., et al. 2008, [arXiv:0806.3968]

Mainieri, V., Hasinger, G., Cappelluti, N., et al. 2007, ApJS, 172, 368

Mainieri, V., et al. 2009, A\&A, in preparation

Mateos, S., et al. 2008, [arXiv: 0809.1939]

McCracken, H. J., Peacock, J. A., Guzzo, L., et al. 2007, ApJS, 172, 314

Moretti, A., Campana, S., Lazzati, D., \& Tagliaferri, G. 2003, ApJ, 588, 696

Narsky, I. 2000, Nucl. Instr. Meth. Phys. Res. A, 450, 444

Page, M. J., Stevens, J. A., Ivison, R. J., \& Carrera, F. J. 2004, ApJ, 611, L85

Panessa, F., \& Bassani, L. 2002, A\&A, 394, 435

Perola, G. C., Puccetti, S., Fiore, F., et al. 2004, A\&A, 421, 491

Predehl, P., Hasinger, G., Böhringer, H., et al. 2006, Proc. SPIE, 6266

Puccetti, S., Fiore, F., D'Elia, V., et al. 2006, A\&A, 457, 501

Ranalli, P., Comastri, A., \& Setti, G. 2003, A\&A, 399, 39

Rosati, P., Giacconi, R., Gilli, R., et al. 2002, ApJ, 566, 667

Salvato, M., et al. 2008, [arXiv:0809.2098]

Scoville, N., Aussel, H., Brusa, M., et al. 2007, ApJS, 172, 1

Schmitt, J. H. M. M., \& Maccacaro, T. 1986, ApJ, 310, 334

Szokoly, G. P., Bergeron, J., Hasinger, G., et al. 2004, ApJS, 155, 271

Treister, E., \& Urry, C. M. 2006, ApJ, 652, L79

Trump, J. R., et al. 2008, [arXiv: 0811.3977]

Ueda, Y., Akiyama, M., Ohta, K., \& Miyaji, T. 2003, ApJ, 598, 886

1 Max-Planck-Institut für Extraterrestrische Physik, Postfach 1312, 85741, Garching bei München, Germany

e-mail: cappelluti@mpe.mpg.de

2 University of Maryland, Baltimore County, 1000 Hilltop Circle, Baltimore, MD 21250, USA

3 INAF - Osservatorio Astronomico di Bologna, via Ranzani 1, 40127 Bologna, Italy

4 ASI Science Data Center, via Galileo Galilei, 00044 Frascati Italy

5 Instituto de Astronomia, Universidad Nacional Autonoma de

Mexico-Ensenada Km. 103 Carretera Tijuana-Ensenada, 22860

Ensenada, BC Mexico, Mexico

6 California Institute of Technology, 105-24 Robinson, 1200 East

California Boulevard, Pasadena, CA 91125, USA

7 Dipartimento di Astronomia, Universit'a di Bologna, via Ranzani

1, 40127 Bologna, Italy

8 Harvard-Smithsonian Center for Astrophysics, 60 Garden St, Cambridge, MA 02138, UK

9 INAF - Osservatorio astronomico di Roma, via Frascati 33, 00044 Monteporzio Catone, Italy

10 Department of Physics, Carnegie Mellon University, 5000 Forbes Avenue, Pittsburgh, PA 15213, USA

11 INAF - Osservatorio Astronomico di Brera - via Brera 28, Milan, Italy

12 Space Telescope Science Institute,3700 San Martin Drive, Baltimore, MD 21218, USA

13 ESO, Karl-Schwarschild-Strasse 2, 85748 Garching, Germany

14 Institute of Astronomy, Department of Physics, Eidgenössische Technische Hochschule, ETH Zurich, 8093, Switzerland

15 Department of Physics, Yale University, PO Box 208121, New Haven, CT 06520-8121, USA

16 Center for Astrophysics and Space Sciences, University of California San Diego, Code 0424, 9500 Gilman Drive, La Jolla, CA 92093, USA 